ARTICLE

\title{
The entangled triplet pair state in acene and heteroacene materials
}

Chaw Keong Yong ${ }^{1,2}$, Andrew J. Musser ${ }^{1,3}$, Sam L. Bayliss', Steven Lukman¹, Hiroyuki Tamura4 , Olga Bubnova1, Rawad K. Hallani ${ }^{5}$, Aurélie Meneau ${ }^{1}$, Roland Resel ${ }^{6}$, Munetaka Maruyama7, Shu Hotta ${ }^{7}$, Laura M. Herz ${ }^{8}$, David Beljonne ${ }^{9}$, John E. Anthony ${ }^{5}$, Jenny Clark $^{3} \&$ Henning Sirringhaus ${ }^{1}$

Entanglement of states is one of the most surprising and counter-intuitive consequences of quantum mechanics, with potent applications in cryptography and computing. In organic materials, one particularly significant manifestation is the spin-entangled triplet-pair state, which mediates the spin-conserving fission of one spin-0 singlet exciton into two spin-1 triplet excitons. Despite long theoretical and experimental exploration, the nature of the triplet-pair state and inter-triplet interactions have proved elusive. Here we use a range of organic semiconductors that undergo singlet exciton fission to reveal the photophysical properties of entangled triplet-pair states. We find that the triplet pair is bound with respect to free triplets with an energy that is largely material independent ( $\sim 30 \mathrm{meV}$ ). During its lifetime, the component triplets behave cooperatively as a singlet and emit light through a Herzberg-Teller-type mechanism, resulting in vibronically structured photoluminescence. In photovoltaic blends, charge transfer can occur from the bound triplet pairs with $>100 \%$ photon-to-charge conversion efficiency.

\footnotetext{
${ }^{1}$ Cavendish Laboratory, Optoelectronics Group, University of Cambridge, Madingley Road, J.J. Thomson Avenue, Cambridge CB3 OHE, UK. ${ }^{2}$ Department of Physics, University of California, Berkeley, California 94720, USA. ${ }^{3}$ Department of Physics and Astronomy, The University of Sheffield, Hicks Buildling, Hounsfield Road, Sheffield S3 7RH, UK. ${ }^{4}$ Department of Chemical System Engineering, The University of Tokyo, 7-3-1, Hongo, Bunkyo-ku, Tokyo 113-8656, Japan. ${ }^{5}$ Department of Chemistry, University of Kentucky, Lexington, Kentucky 40506-0055, USA. ${ }^{6}$ Institute of Solid State Physics, Graz University of Technology, Petersgasse 16, 8010 Graz, Austria. ${ }^{7}$ Faculty of Materials Science and Engineering, Kyoto Institute of Technology, Matsugasaki, Sakyo-ku, Kyoto 606-8585, Japan. ${ }^{8}$ Clarendon Laboratory, Department of Physics, University of Oxford, Parks Road, Oxford OX1 3PU, UK. ${ }^{9}$ Laboratory for Chemistry of Novel Materials, University of Mons, Place du Parc 20, B-7000 Mons, Belgium. Correspondence and requests for materials should be addressed to C.K.Y. (email: chawkeong@berkeley.edu) or to J.C. (email: jenny.clark@sheffield.ac.uk) or to H.S. (email: hs220@cam.ac.uk).
} 
T he entangled pair of triplet excitons ${ }^{1}$ (TT) is thought to be essential to the rapid, spin-allowed formation of two triplets from a single absorbed photon ${ }^{1-3}$. Its overall singlet character may allow for surprisingly fast, long-distance triplet exciton transport following singlet fission ${ }^{4}$. The very same state also mediates the reverse of singlet fission: triplet-triplet annihilation (TTA), where two triplets fuse to form an emissive singlet, a process exploited for biomedical imaging 5,6 , high-density secure data storage ${ }^{7}$, incoherent energy up-conversion ${ }^{8}$ and high-efficiency blue organic light-emitting diodes ${ }^{9}$. Beyond applications of singlet fission and TTA, a clearer understanding of the ${ }^{1}$ (TT) state will shed light on the nature of the interaction between triplet excitons and may open the way to new physics involving entangled bosons ${ }^{10,11}$. Despite significant theoretical effor ${ }^{10-20}$, few experiments have succeeded in directly probing the ${ }^{1}(\mathrm{TT})$ state in any system ${ }^{21-25}$, particularly in solid films relevant to eventual singlet fission devices. We are aware of a recent study of the ${ }^{1}$ (TT) state in solid films of 6,13Bis(triisopropylsilylethynyl) (TIPS)-tetracene, which crystallizes into a structure unique among singlet fission materials ${ }^{26}$. The nature of this state and its role in singlet fission in the wider range of acene and heteroacene singletfission materials are not clear, nor its importance in the physics of singlet fission-based solar cells. During the review process, several other reports have emerged describing properties of triplet-pair states, including dynamic equilibrium with $S_{1}$ (ref. 27), the formation of quintet-coupled triplet pairs ${ }^{28,29}$, ultrafast formation in a strongly exothermic system ${ }^{30}$ and theoretical re-evaluations of triplet-pair interactions ${ }^{31-33}$. These works highlight the ongoing debate about the nature of ${ }^{1}(\mathrm{TT})$. Of those that discuss energetics, all consider ${ }^{1}$ (TT) to be higher in energy than $T_{1}+T_{1}$. Here we demonstrate this is incorrect and show that ${ }^{1}(\mathrm{TT})$ is bound with respect to free triplets.

Here we probe the ${ }^{1}$ (TT) state experimentally and theoretically in a range of acene and heteroacene materials (Fig. 1a). We demonstrate the generality of the presence of a bound ${ }^{1}$ (TT) state as the immediate product of singlet fission. The processes of formation and decay of ${ }^{1}(\mathrm{TT})$ are shown in Fig. 1b, a schematic inspired by Tayebjee et al. ${ }^{34}$ and references therein 13,35 . Formation of ${ }^{1}(\mathrm{TT})$ is temperature-independent in polycrystalline films and is thought to occur via a combination of intra- and intermolecular motion from the photoexcited singlet $^{12-14,21,34,36,37}$. Once formed, ${ }^{1}(\mathrm{TT})$ can undergo one of three detectable decay processes as follows: (i) form free (unbound) triplets, a process aided by disorder at low temperatures and thermally activated above $50 \mathrm{~K}$; (ii) emit (red arrows) by way of symmetry-breaking distortions enabling intensity borrowing from nearby bright states; and (iii) with sufficient energy, 'back transfer' to $S_{1}$ leading to delayed fluorescence. We also infer that a significant proportion of ${ }^{1}$ (TT) states undergo non-radiative decay, presumably directly to the ground state. In photovoltaic blends, we find that charge transfer from this bound, entangled triplet pair is as efficient as that from free triplets giving photon-to-charge conversion quantum efficiencies of $>100 \%$.

\section{Results}

Emissive triplet-pair state. Figure la shows the chemical structures of the molecules used. All undergo singlet fission with time constants ranging from sub- $100 \mathrm{fs}$ in pentacene and TIPS-pentacene to $50-300 \mathrm{ps}$ in 2,8-difluoro-5,11-bis(triethylsilylethynyl)anthradithiophene $\left(\mathrm{F}_{2}\right.$-TES $\left.\mathrm{ADT}\right)$, rubrene and tetracene ${ }^{15,38-40}$. The time-integrated, temperaturedependent photoluminescence (PL) spectra of $\mathrm{F}_{2}$-TES ADT are shown in Fig. 1c. Similar to tetracene films, described in refs $34,41-43$, emission at temperatures between 200 and $90 \mathrm{~K}$ is dominated by a new peak, red-shifted from the $0-0$ emission peak. Below $90 \mathrm{~K}$, an enhanced $0-0$ peak once again dominates the spectrum. Given the similarity in temperature-dependent emission and, as we demonstrate below, singlet fission dynamics in $\mathrm{F}_{2}$-TES ADT and tetracene, we suggest a similar origin for the red-shifted emissive species. We propose in the following that this emission arises from a bound, entangled triplet pair, ${ }^{1}(\mathrm{TT})$, the immediate product of singlet fission.

To better understand the origin of the PL spectral shifts, we performed time-resolved measurements in $\mathrm{F}_{2}$-TES ADT solutions (Fig. 2a) and thin films (Fig. 2b). We chose to focus on $\mathrm{F}_{2}$-TES ADT as, unlike tetracene, $F_{2}$-TES ADT shows no significant structural phase transition in the thin films (Supplementary Fig. 4), no evidence of 'excimer' emission ${ }^{43,44}$ and strong triplet features in transient absorption (TA) spectra. In dilute solution only singlet states emit, with a lifetime of $12 \mathrm{~ns}$. Concentrated solutions and thin films, however, show evidence of multiple emitting species. To separate spectral features with correlated decay kinetics we use a spectral decomposition method based on a genetic algorithm ${ }^{45}$ (see the Methods section for more details). The PL maps are best modelled as a sum of two independent species (solid lines) both in concentrated solution and thin films. The same procedure was applied to data taken at other temperatures (Supplementary Fig. 5), yielding clearly delineated prompt and delayed PL spectra (Fig. 2c).

In concentrated solution, the singlet converts into a red-shifted species maintaining vibronic structure. Likewise, in solid films decomposition reveals correlation between prompt and delayed emitter kinetics: the delayed species grows at the expense of the prompt. Although the 'prompt' emitter lifetime remains essentially unchanged over the temperature range, the 'delayed' emitter lifetime increases substantially with decreasing temperature. Conversely, the prompt emission spectrum changes dramatically with temperature. The enhancement of the $0-0$ peak, a property common to materials such as tetracene, which show super-radiance at low temperature ${ }^{46}$, allows assignment to the singlet exciton. The delayed red-shifted emission spectrum, which dominates $\mathrm{F}_{2}$-TES ADT film emission at intermediate temperatures, is remarkably constant below $200 \mathrm{~K}$ and can be assigned to ${ }^{1}(\mathrm{TT})$, see below. At higher temperatures, the increased intensity in the delayed component at $585 \mathrm{~nm}$, co-incident with the singlet $0-0$ emission, suggests thermally activated delayed fluorescence with the same lifetime as ${ }^{1}(\mathrm{TT})$ (process (iii) in Fig. 1b).

Spin-entanglement of ${ }^{1}(\mathrm{TT})$ observed by quantum beating. Interestingly, following femtosecond excitation at $300 \mathrm{~K}$ we observed temporal oscillations of the PL, as shown in Fig. 3a. Similar to tetracene ${ }^{47,48}$ (right panel), the amplitude reduces gradually within an $8 \mathrm{~ns}$ window and follows the extracted delayed emitter kinetics. The Fourier transform reveals the existence of three beat frequencies (Fig. $3 b$ ) at 1.1, 2.0 and $2.9 \pm 0.1 \mathrm{GHz}$ that are strikingly similar to those in tetracene (1.0, 1.9 and $3.1 \pm 0.1 \mathrm{GHz}$, respectively), as discussed in Supplementary Note 1 . These quantum beats have never been reported in other materials than tetracene, and are a manifestation of the spin properties of triplet pairs following singlet fission ${ }^{47,48}$. Briefly, in the simplest two-electron two-hole picture of singlet fission, there are 16 possible combinations of 4 spins: 2 singlets, 9 triplets and 5 quintets. Singlet fission entails spin-allowed conversion between the two singlet states, one of which is a superposition of triplet-pair states of the form $|S\rangle=\frac{1}{\sqrt{3}}(|X X\rangle+|Y Y\rangle+|Z Z\rangle)$ in the zero-field basis (see 
a
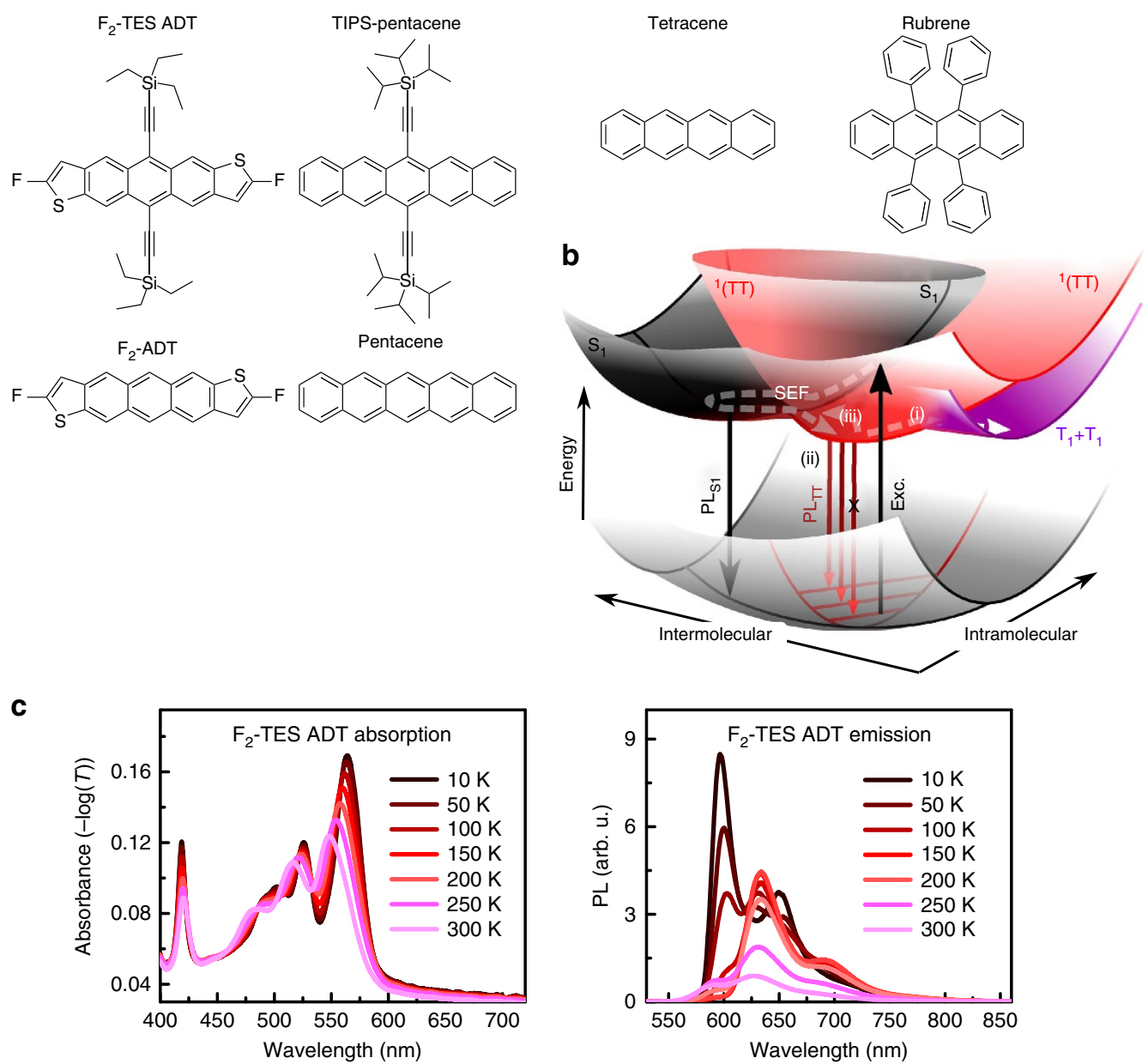

Figure 1 | Singlet exciton fission in polyacenes. (a) Molecules investigated in this study. (b) Schematic potential energy surface denoting key photophysical processes (dashed arrows) in singlet fission materials. Photoexcitation (Exc.) is followed by singlet (black) relaxation along inter- and intramolecular coordinates, resulting in the observed Stokes shift $\left(\mathrm{PL}_{\mathrm{S1}}\right)$. Further relaxation into the bound, spin-coherent triplet pair state ${ }^{1}(\mathrm{TT})$ (red) constitutes singlet exciton fission (SEF). Three detectable decay processes are possible from ${ }^{1}(\mathrm{TT})$. (i) Thermally activated dissociation into free triplets (purple), which is aided by disorder/grain boundaries and exhibits a typical activation energy of 20-40 meV. (ii) Direct 'delayed' emission from ${ }^{1}\left(\mathrm{TTT}_{\mathrm{T}}\right.$ ( $\mathrm{PL}_{\mathrm{TT}}$ ) through Herzberg-Teller intensity borrowing. As a consequence of this mechanism, the 0-0 transition is suppressed. (iii) Thermally activated back-transfer

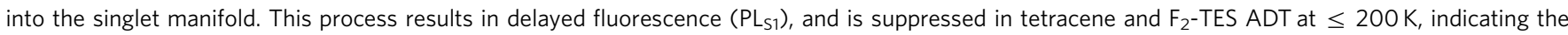
presence of a slight energy barrier. (c) Temperature-dependent absorption and PL spectra of $F_{2}$-TES ADT films.

Supplementary Notes 1 and 2 for details including magnetic resonance characterization (Supplementary Fig. 6) and details relating to quantum beating (Supplementary Fig. 7)). $|S\rangle$ is not an energy eigenstate of the system and $|X X\rangle,|Y Y\rangle$ and $|Z Z\rangle$ acquire relative phases, evolving in time with frequencies governed by their energy spacing. As the state periodically approaches its initial composition (that is, ${ }^{1}(\mathrm{TT})$ ), there is significant enhancement of the rate to re-form the original, fluorescent singlet through TTA if this is energetically allowed. The observed PL quantum beats thus reveal modulation of the radiative TTA probability due to spin conservation. The oscillation amplitude follows the extracted delayed PL kinetics in $\mathrm{F}_{2}$-TES ADT and tetracene, clearly linking the delayed emission to the ${ }^{1}(\mathrm{TT})$ state generated by singlet fission. The beats' persistence over the ${ }^{1}(\mathrm{TT})$ lifetime indicates that spin coherence is maintained over at least that timescale, and that ${ }^{1}$ (TT) is thus an entangled triplet-pair state.

${ }^{1}$ (TT) emission through Herzberg-Teller symmetry breaking. It is unexpected for this doubly excited state $\left({ }^{1}(\mathrm{TT})\right)$ to emit, especially with the well-defined vibronic progression seen in
Fig. 2c, right panel. To probe the origin of this behaviour we have performed $a b$ initio $(\operatorname{CASSCF}(4,4))$ excited-state calculations on $\pi$-stacked dimers in the crystal geometry (details in the Methods section below and Table 1). Mixing with charge transfer and singlet states stabilizes ${ }^{1}$ (TT) relative to the singlet and non-interacting triplet pairs. Importantly, the bright singlet contribution to the adiabatic ${ }^{1}$ (TT) state vanishes at the equilibrium geometry ${ }^{16,20}{ }^{1}$ (TT) instead mixes only with a dark singlet, with emission thus forbidden. Geometric distortion activates slight mixing with the bright state, resulting in finite but low radiative rate (that is, $\sim 40 \times$ lower than that of the initial singlet state in $\mathrm{F}_{2}$-TES ADT at $50 \mathrm{~K}$ ). Nonetheless, the ${ }^{1}(\mathrm{TT})$ emission can dominate the spectrum at intermediate temperatures due to its long lifetime. This mechanism is equivalent to Herzberg-Teller coupling ${ }^{49}$, in which a symmetry-forbidden dark state can couple to a vibration of the bright-state symmetry and thereby obtain the correct symmetry for mixing or intensity borrowing from nearby bright states.

We propose that this mechanism explains the vibronically structured red-shifted PL seen in many acenes in the literature. 

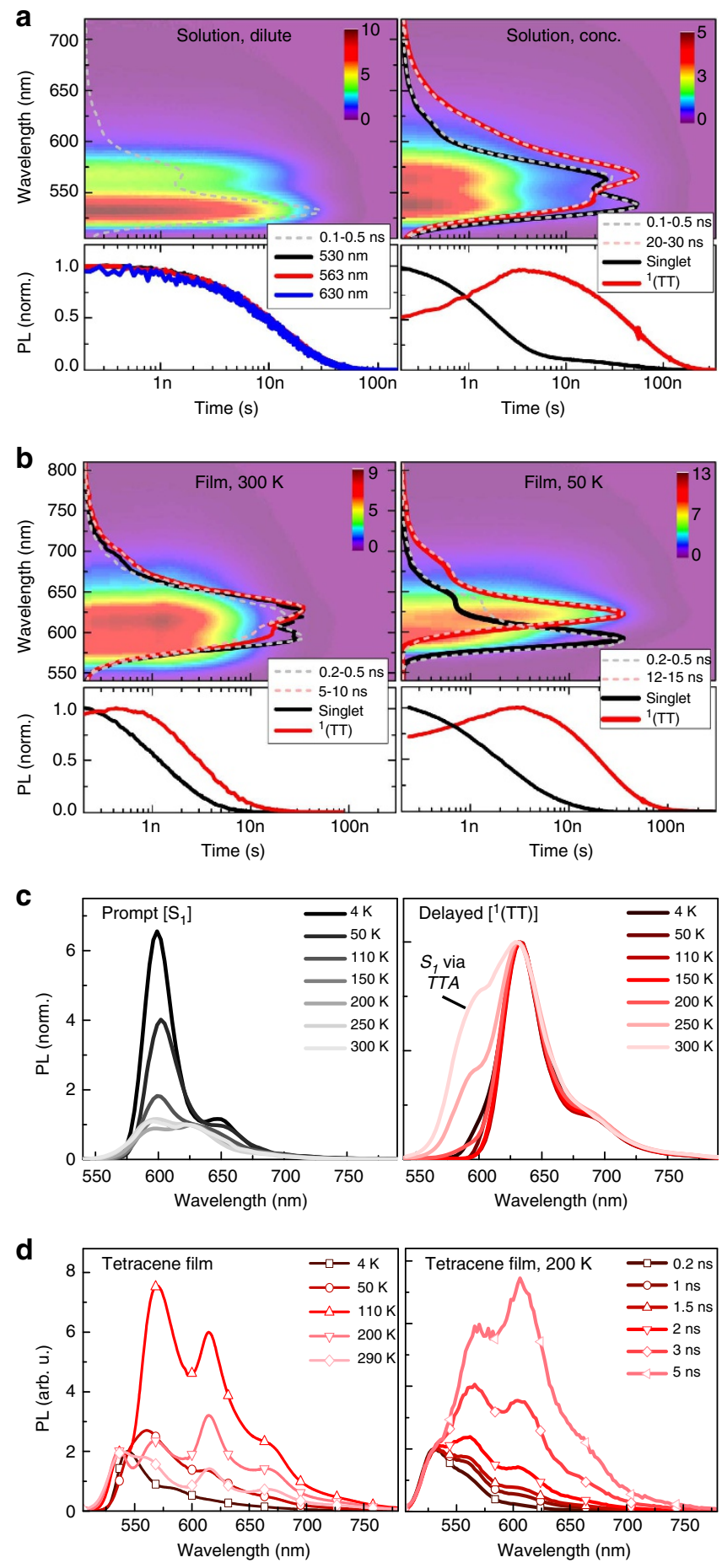

Figure $\left.2\right|^{\mathbf{1}}$ (TT) emission. $F_{2}$-TES ADT time-resolved PL spectral maps for (a) solutions and (b) thin films. Prompt and delayed spectral components (solid lines) were extracted as described in the main text. Dashed lines are normalized spectral cuts from raw PL at the indicated time delays. Associated population kinetics (bottom) reveal sequential conversion from prompt to delayed emission. (c) Prompt and delayed $\mathrm{F}_{2}$ TES ADT film spectra extracted over the full temperature range, attributed to $S_{1}$ and ${ }^{1}(T T)$, respectively. The delayed $\mathrm{PL}$ broadening at $>200 \mathrm{~K}$ reveals thermal repopulation of $\mathrm{S}_{1}$ from ${ }^{1}(\mathrm{TT})$ via TTA, process (iii) in Fig. 1b. (d) Normalized PL spectra of a tetracene film as a function of temperature in steady-state conditions (left) and time after excitation at $200 \mathrm{~K}$ (right).
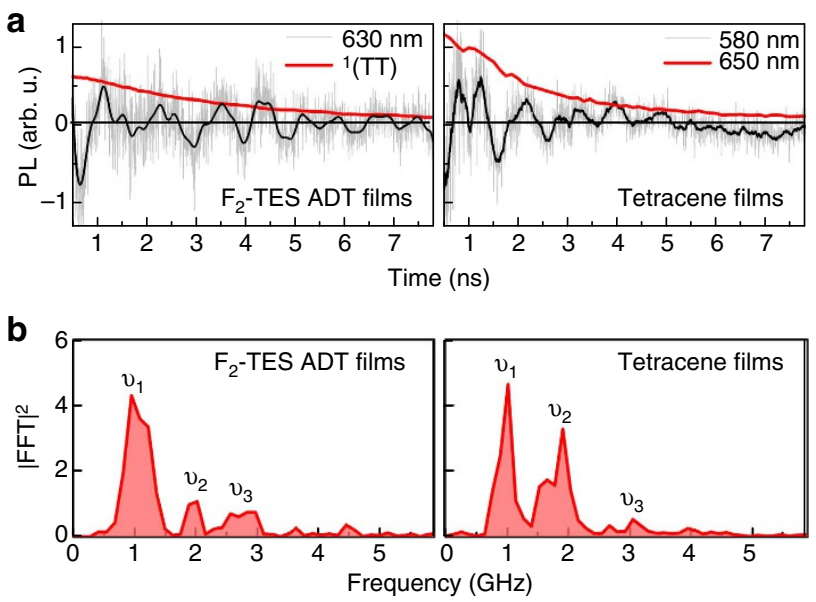

Figure 3 | PL quantum beating from spin-entangled ${ }^{\mathbf{1}}$ (TT). (a) PL decay of $\mathrm{F}_{2}$-TES ADT and tetracene films following femtosecond excitation at $300 \mathrm{~K}$, after subtraction of multi-exponential envelope. Resulting slow oscillations (grey: raw, black: smoothed) decay in agreement with extracted ${ }^{1}$ (TT) kinetics in $F_{2}$-TES ADT and red-emitting feature in tetracene (red). (b) Fourier transform of the oscillations, revealing quantum beats at 1.05, $1.96,2.88 \pm 0.1 \mathrm{GHz}\left(F_{2}\right.$-TES ADT) and $0.99,1.91$ and $3.06 \pm 0.1 \mathrm{GHz}$ (tetracene).

As discussed above, tetracene demonstrates very similar temperature-dependent $\mathrm{PL}$ to $\mathrm{F}_{2}$-TES ADT (Fig. 2d, Supplementary Note 3, Supplementary Figs 8 and 9, and refs $34,41-43)$, with a red-shifted delayed component ${ }^{34,42}$ that decays with the same lifetime as the quantum beating (Fig. 3a). Even on much longer timescales, structured ${ }^{1}(\mathrm{TT})$ emission may be preferentially regenerated by annihilation from 'trapped' triplets due to its lower electronic energy ${ }^{34,50}$. Rubrene (Supplementary Fig. 10) likewise shows evidence of a temperature-dependent long-lived PL feature, somewhat obscured by the similarity of the $S_{1}$ and ${ }^{1}(\mathrm{TT})$ energies; even pentacene has been shown to demonstrate similarly shaped emission in films $\mathrm{s}^{51}$ and crystals ${ }^{52}$. Under Herzberg-Teller coupling the $0-0$ peak is suppressed ${ }^{53}$; thus, we assign the most prominent feature in the ${ }^{1}(\mathrm{TT})$ spectrum to the $0-1$ peak, akin to emission from the 'triplet-pair'-like ${ }^{54} S_{1}$ state in polyenes ${ }^{55}$. Following this model, we determine the energy of the ${ }^{1}$ (TT) $0-0$ peak from the spacing between observed $0-1$ and $0-2$ peaks (Supplementary Fig. 1). We find that the energy extracted from PL scales with the expected $2 \times T_{1}$ energy from independent measurements of the triplet energy, with an offset which could account for the binding energy of the triplets in ${ }^{1}(\mathrm{TT})$. This is shown in Fig. 4.

This model has critical implications for singlet fission energetics in 'slow' tetracene-like materials. In $\mathrm{F}_{2}$-TES ADT and similar materials, ${ }^{1}$ (TT) and $S_{1}$ are roughly isoenergetic. Indeed, the delayed spectra in Fig. $2 c$ reveal temperature-dependent broadening consistent with delayed singlet re-formation, which is turned off $<200 \mathrm{~K}$. The implicit small barrier to singlet re-formation can only be reconciled with the observed ${ }^{1}$ (TT) PL spectrum through the Herzberg-Teller model. The ability of ${ }^{1}$ (TT) to emit and the spin-conservation considerations above suggest that quantum beating could likewise be anticipated in the direct ${ }^{1}(\mathrm{TT})$ emission, suggesting a need for spectrally resolved quantum beating studies.

Identification of ${ }^{1}$ (TT) in TA spectroscopy. For further insight into the formation and decay of ${ }^{1}$ (TT) we used TA spectroscopy. Figure $5 \mathrm{a}$ shows the TA spectra of $\mathrm{F}_{2}$-TES ADT films collected at $300 \mathrm{~K}$ and we can immediately distinguish two primary species: 


\begin{tabular}{|c|c|c|c|}
\hline & Bright $S_{1}$ & Dark $\mathbf{S}_{\mathbf{1}}$ & CT \\
\hline TIPS-Pn (T T equilibrium) & 0.00 & 0.28 & 1.34 \\
\hline $\mathrm{F}_{2}$-TES ADT (T Tequilibrium) & 0.00 & 0.24 & 0.96 \\
\hline$F_{2}$-TES ADT (T G equilibrium) & 0.46 & 0.02 & 0.08 \\
\hline
\end{tabular}

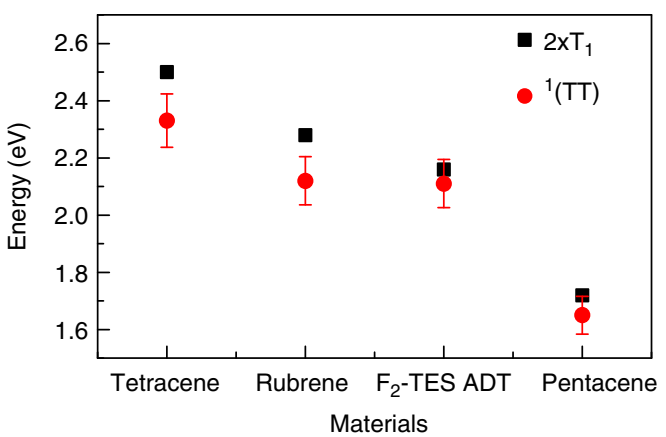

Figure $\left.4\right|^{1}$ (TT) energies. ${ }^{1}(\mathrm{TT})$ and $2 \times \mathrm{T}_{1}$ energies for materials studied here: ${ }^{1}(\mathrm{TT})$ energy extracted from PL spectra, see Supplementary Fig. $1, \mathrm{~T}_{1}$ from phosphorescence measurements ( $F_{2}-T E S$ ADT, rubrene Supplementary Figs 2,3 ) and literature ${ }^{61,73}$. Error bars reflect uncertainty in fitting the ${ }^{1}(\mathrm{TT})$ vibronic progression.

the initially formed singlet exciton and a subsequent long-lived photo-induced absorption (PIA) band centred at $970 \mathrm{~nm}$. To aid assignment of this feature, we examine $\mathrm{F}_{2}$-TES ADT solutions at several concentrations $\mathrm{s}^{21,36}$ (Fig. 5b,c). For simplicity, we focus on the probe range $800-1,100 \mathrm{~nm}$, where all states of interest have distinct PIA features (full characterization in Supplementary Figs 5, 11-13). In dilute solutions (Fig. 5b,c top), we observe $\sim 12$ ns singlet decay consistent with measured PL (Fig. 2a). Concentrated solutions ( $32 \mathrm{mM}$, Fig. $5 \mathrm{~b}, \mathrm{c}$ middle) exhibit faster singlet decay and spectral evolution over 100s of nanoseconds to yield long-lived triplets $\left(T_{1}+T_{1} ; \quad\right.$ sensitization $^{56}$ in Supplementary Fig. 11). Between singlet and triplet, we observe an intermediate, which decays with the same kinetics (red trace) as the delayed ${ }^{1}(\mathrm{TT})$ emission (pink dashed trace), enabling assignment to ${ }^{1}(\mathrm{TT})$. The last panels in Fig. 5b,c reveal a similar progression in thin films at $50 \mathrm{~K}$ (Supplementary Fig. 13 for other temperatures), demonstrating that ${ }^{1}(\mathrm{TT})$ acts as a distinct singlet fission intermediate even in the solid state. We expect the ${ }^{1}$ (TT) PIA absorption cross-section to be similar to that of two free triplets. Therefore, the reduction in signal from 3-100 ns in the raw TA kinetic (pink trace in Fig. 5c) suggests significant decay without forming free triplets. The total PL quantum yield of $10 \%$ at $50 \mathrm{~K}$ indicates that this decay is largely non-radiative.

We observe the same progression in rubrene, TIPS-pentacene and pentacene (Fig. 6), confirming the presence of the ${ }^{1}(\mathrm{TT})$ intermediate in all three (see also Supplementary Figs 14-18). In rubrene, these features match the red-shifted delayed PL kinetics (Supplementary Fig. 10). No PL was detected in TIPS-pentacene and pentacene films. We performed similar measurements on polycrystalline tetracene films (Supplementary Fig. 19) but, as discussed in Supplementary Note 3, find that these are difficult due to small triplet absorption cross-sections ${ }^{43,57-59}$, coupled with the need for low excitation density to avoid exciton-exciton annihilation $^{60}$. In the other materials (Supplementary Fig. 20),
${ }^{1}(\mathrm{TT})$ decay was completely independent of intensity, indicating that any recombination is geminate. By contrast, free triplets exhibit fluence-dependent recombination. Interestingly, our results show that $S_{1}$ decay and ${ }^{1}(\mathrm{TT})$ growth are largely temperature-independent (Fig. 5 and Supplementary Figs 13 and 14), even in rubrene ${ }^{39}$.

Stabilization of ${ }^{1}(\mathrm{TT})$ versus $S_{1}$ and $2 \times T_{1}$. A temperature dependence does, however, appear in the subsequent process, in which the bound triplet pair separates into free triplet excitons (Fig. 5 and Supplementary Fig. 13). It is noteworthy that this is even observed in TIPS-pentacene and pentacene (Supplementary Note 4 and Supplementary Figs 16 and 18), where the overall process of singlet fission is exothermic (Supplementary Fig. 21 and ref. 61). In Fig. 7a we plot ${ }^{1}$ (TT) and free triplet population dynamics for $\mathrm{F}_{2}$-TES ADT films from 4-250 K. Data for other molecules can be found in Supplementary Fig. 20 ( $\mathrm{F}_{2}$ ADT single crystal), Supplementary Fig. 13 (rubrene), Supplementary Fig. 15 (TIPS-pentacene) and Supplementary Fig. 17 (pentacene). From an Arrhenius fit to the corresponding rates of free triplet formation at elevated temperature, we obtain a general phenomenological activation barrier $\sim 20-40 \mathrm{meV}$ (Fig. 7b), although the underlying rates vary by nearly two orders of magnitude. These results demonstrate that ${ }^{1}(\mathrm{TT})$ is stabilized with respect not only to the initial singlet but also to two free triplet excitons, confirming its bound nature ${ }^{21}$. We consider that this behaviour is general to the acenes and is likely to be a common property of all singlet fission materials, recalling the well-known stabilization of the triplet-pair $2 \mathrm{~A}_{\mathrm{g}}$ state in polyenes relative to two free triplets ${ }^{54,56,62}$.

To better understand this binding and thermally activated decay, we have analysed conversion from ${ }^{1}$ (TT) into free triplets based on Marcus-Levich-Jortner rate theory parameterized against $a b$ initio calculations (Table 2 and Fig. $7 \mathrm{c}$, full details in the Methods section). We considered the $\pi$-stacked crystal geometries of $\mathrm{F}_{2}$-TES ADT, TIPS-pentacene and rubrene, in which separation is one dimensional. The enthalpy change $\Delta H$ for separation was determined using trimer models in which molecules adopted either the fully relaxed triplet geometry $(T)$ or the ground-state geometry $(G)$. Bound and separated triplet pairs then correspond to TTG and TGT configurations, respectively. The free energy stabilization going from TGT to TTG completely dominates the activation energy $\Delta E$. The stabilization energies are overestimated by these calculations, probably due to the presence of polymorphism and the assumed single crystal geometry. For instance, we calculate that increasing the $\pi$-stack distance in TIPS-pentacene by $0.1 \AA$ decreases the $\Delta H$ by $\sim 30 \%$. Nonetheless, the predicted trends in the $\Delta E$ values match well with measurements.

Moreover, the magnitudes of $\Delta H(50-100 \mathrm{meV})$ correlate well with the admixture of singlet and charge-transfer configurations (Table 1), indicating that this mixing is a primary driver of ${ }^{1}(\mathrm{TT})$ stabilization, although orbital delocalization also contributes. The 

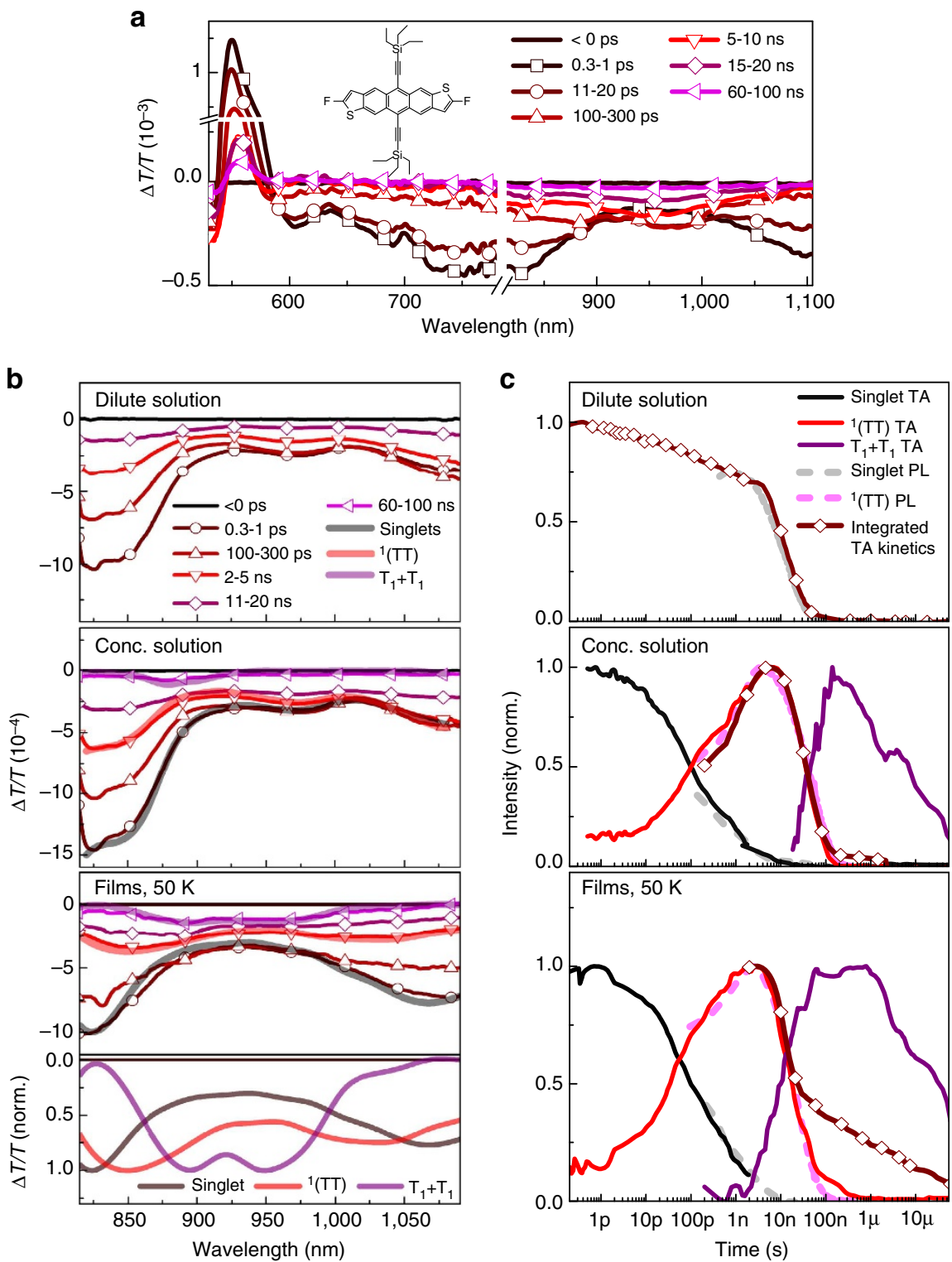

Figure $\mathbf{5}$ | $\mathbf{1}(\mathbf{T T})$ absorption in $\mathbf{F}_{\mathbf{2}}$-TES ADT. (a) TA spectra of $\mathrm{F}_{2}$-TES ADT films at $300 \mathrm{~K}$, averaged over the indicated delay ranges. The initial singlet state (peaks $750-800 \mathrm{~nm}$ and $>1,100 \mathrm{~nm}$ ) converts directly into triplet excitons (peak centred at $970 \mathrm{~nm}$ ) over $\sim 300 \mathrm{ps}$, through singlet fission. (b) TA spectra of $\mathrm{F}_{2}$-TES ADT dilute solution (top, $0.08 \mathrm{mM}$ ), concentrated solution (middle, $32 \mathrm{mM}$ ) and thin film measured at $50 \mathrm{~K}$ (bottom). Extracted spectra (thick lines) closely follow the raw data at different time delays, revealing the presence of substantial triplet population $\left(T_{1}+T_{1}\right)$ in concentrated solutions and thin film, as well as a distinct intermediate state ${ }^{1}(\mathrm{TT})$. Extracted spectra of the three species in the film at $50 \mathrm{~K}$ are normalized in the bottom panel for clarity. (c) Normalized population kinetics of excited state species extracted from TA maps, exhibiting a sequential progression: singlet (black), ${ }^{1}$ (TT) (red) and free triplets (purple). Also plotted are the integrated NIR TA signal (brown, diamonds) and kinetics extracted from the separate transient PL experiment (dashed lines). The agreement of PL and TA population kinetics confirms the same species are observed in both experiments.

electronic coupling for intermolecular triplet transfer $V_{\mathrm{T}-\mathrm{T}}$ was estimated using $\pi$-stacked dimer models and agrees well with previous studies $^{38}$.

As shown in Fig. 7d, the resulting rate equation yields a strong temperature dependence for ${ }^{1}$ (TT) separation, in good agreement with experimental results given the simple model. Importantly, we reproduce the large rate difference between TIPS-pentacene and $\mathrm{F}_{2}$-TES ADT, and the smaller difference between $\mathrm{F}_{2}$-TES ADT and rubrene. These differences cannot be attributed to $\Delta H$ (comparable for all three) and instead follow $V_{\mathrm{T}-\mathrm{T}}$. Thus, the underlying rate for ${ }^{1}(\mathrm{TT})$ separation is governed by the intrinsic triplet mobility. At the same time, we note that the overall activation energy is dominated by $\Delta H$ rather than the inter- or intramolecular reorganization energies. We can thus conclude that the measured high-temperature activation energies reflect the energy stabilization of bound versus free triplet pairs, and that triplet-pair separation can be described in terms of triplet hopping from the bound state. The most significant discrepancy between theory and experiment is at low temperature: the model predicts vanishingly small ${ }^{1}(\mathrm{TT})$ separation, whereas we measure very little change in the rate from $\sim 50 \mathrm{~K}$ to lower temperatures. We propose that structural and/or energetic disorder, for instance as would be found at grain boundaries, accounts for the persistence of low-barrier free triplet formation.

To verify this, we have performed similar detailed TA and PL measurements on single crystals of difluorinated anthradithiophene $\left(\mathrm{F}_{2}-\mathrm{ADT}\right.$, Fig. $7 \mathrm{~d}, \mathrm{e}$ and Supplementary Fig. 20). We observed similar singlet and ${ }^{1}(\mathrm{TT})$ emission, 
a

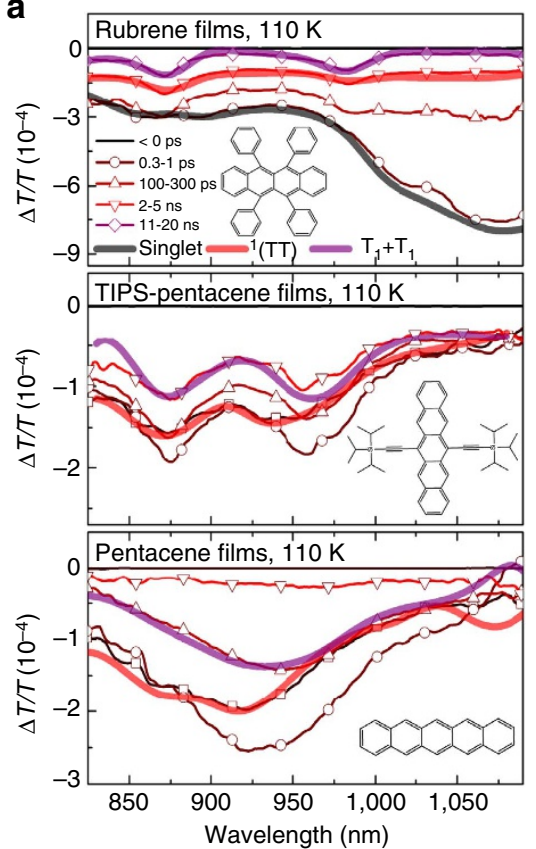

b

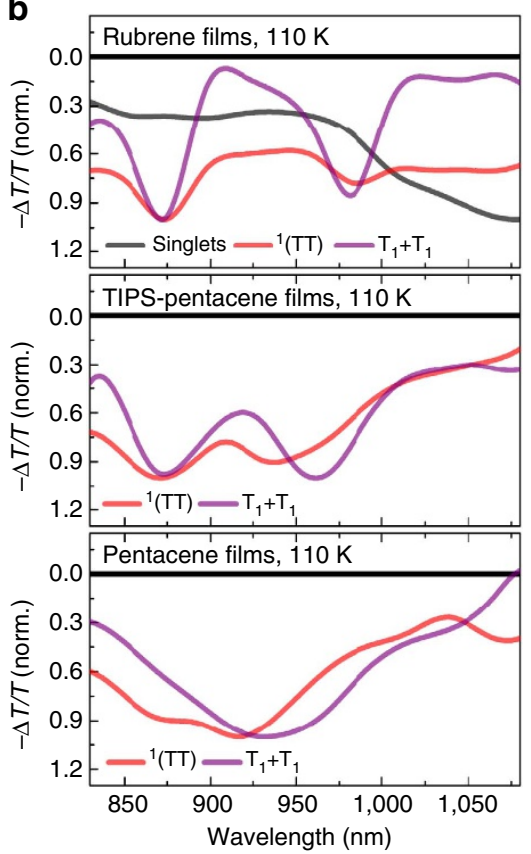

C
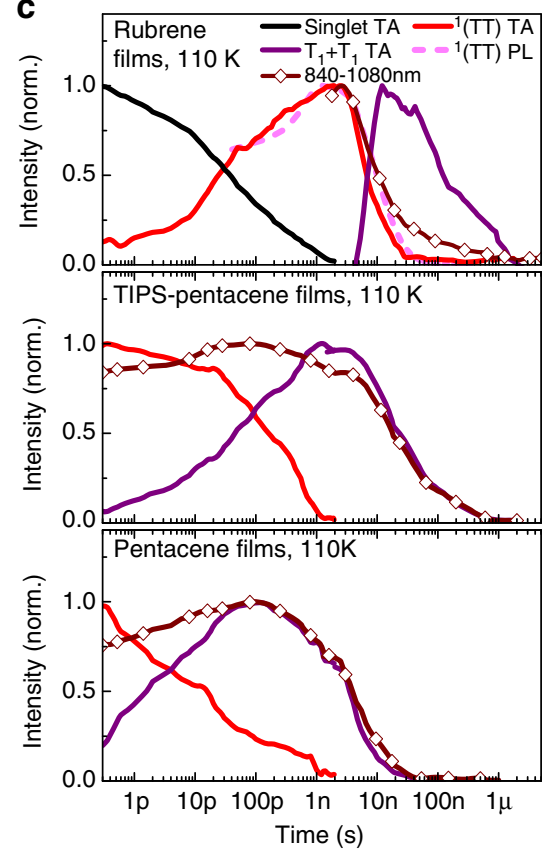

Figure 6 | 1(TT)-mediated singlet fission in polyacenes. (a) TA spectra of rubrene, TIPS-pentacene and pentacene thin films measured at low temperature, averaged over the indicated delay ranges (thin lines). Spectral decomposition analysis (thick lines) provides spectra of singlet (black), ${ }^{1}$ (TT) (red) and free triplet (purple). Note the rate of singlet fission in TIPS-pentacene and pentacene is too fast to clearly isolate singlet spectral features. In all materials, the conversion from ${ }^{1}$ (TT) to free triplets can be identified from peak shifts in the PIA. (b) Extracted singlet, ${ }^{1}(\mathrm{TT})$ and free triplet spectra from (a) normalized for clarity. (c) Normalized population kinetics of excited state species extracted from TA maps, along with integrated raw TA signal (brown, diamonds) and extracted PL kinetics, where applicable (dashed). All materials exhibit the same sequential model.

with clear temperature dependence of the ${ }^{1}(\mathrm{TT})$ lifetime above $200 \mathrm{~K}$ but little temperature dependence below. In TA, we found free triplet formation at $\geq 200 \mathrm{~K}$ but observed no free triplets at lower temperature. These results support our model for the role of disorder: in the absence of structural or energetic disorder, the ${ }^{1}$ (TT) state must be separated through thermal energy alone, leading to complete suppression of the process at low temperature.

Concerted two-electron transfer from ${ }^{1}(\mathrm{TT})$ in solar cells. The low rate for ${ }^{1}(\mathrm{TT})$ separation in $\mathrm{F}_{2}$-TES ADT, tetracene $\mathrm{e}^{47}$ and rubrene suggests that this state may play a significant role in fission-sensitized solar cells, unlike in pentacene-based devices where the extrapolated room-temperature ${ }^{1}(\mathrm{TT})$ lifetime is only $\sim 200 \mathrm{fs}$ (ref. 22). To elucidate the behaviour of ${ }^{1}$ (TT) in photovoltaic blend films and devices, we have studied bulk-heterojunction films of $\mathrm{F}_{2}$-TES ADT and [6,6]-phenyl C71 butyric acid methyl ester $\left(\mathrm{PC}_{71} \mathrm{BM}\right)$ in 4:1 molar ratio, in which we expect significant demixing. The device structure is shown schematically in Fig. 8a with electrical characteristics in Fig. 8b,c. We focus here on two representative temperatures: 300 and $50 \mathrm{~K}$, where the primary long-lived species in $\mathrm{F}_{2}$-TES ADT should either be free or bound spin-entangled triplets, respectively (for other temperatures, see Supplementary Fig. 22). As noted above, the yield of free triplets relative to ${ }^{1}$ (TT) drops as the temperature is lowered (Fig. 8f).

Figure 8 d,e shows that at $300 \mathrm{~K}$ free triplets are formed, revealing that a significant proportion of $\mathrm{F}_{2}$-TES ADT molecules must be far from the fullerene interface. Spectral decomposition (Fig. 8d) reveals a third species, identified as a hole polaron (radical cation) in $\mathrm{F}_{2}$-TES ADT from comparison with charge-modulation spectroscopy (dashed line in Fig. 8d and Supplementary Fig. 23). Charge-modulation spectroscopy is used to determine the optical spectra and absorption cross-section of hole polarons in the solidstate (see Supplementary Note 5 for details). From the strength of the hole polaron contribution to the TA signal we can then directly extract the charge population formed (see Supplementary Note 5 and Supplementary Table 1), resulting in a photon-to-charge quantum yield of $\sim 120 \%$. Equivalent device characterization in Fig. $8 \mathrm{~b}, \mathrm{c}$ shows that, although the power conversion efficiency of the device is low $(1.7 \%)$, the peak internal quantum efficiency approached $120 \%$ in the spectral range of $\mathrm{F}_{2}$-TES ADT (Fig. $8 \mathrm{c}$ ) in excellent agreement with the TA results.

The $300 \mathrm{~K}$ extracted kinetics shown in Fig. 8e reveal that these charges form at the expense of both bound and free triplet excitons, resulting in a reduction of both the ${ }^{1}(\mathrm{TT}) \mathrm{PL}$ and free triplet TA lifetimes. There is thus a competition between two modes of ${ }^{1}(\mathrm{TT})$ decay: diffusion to a $\mathrm{PC}_{71} \mathrm{BM}$ interface with direct charge generation, and thermally activated separation into free triplets, which in turn diffuse to the interface to form charges.

At $50 \mathrm{~K}$, the contribution of ${ }^{1}(\mathrm{TT})$ to charge formation can be isolated, as free triplet formation is strongly suppressed. Indeed, we find that the relative yield of free triplets is $>50 \%$ lower at $50 \mathrm{~K}$ than at $300 \mathrm{~K}$ (Fig. 8f). In the TA dynamics, following formation of bound triplet pairs, we observe separation into charges with no sign of intermediate free triplets. Intriguingly, we find that the ${ }^{1}$ (TT) lifetime in neat and blend films is strikingly similar at $50 \mathrm{~K}$. We can only reconcile this by citing the same rate-determining process for both ${ }^{1}$ (TT) separation to free triplets and charge separation from ${ }^{1}(\mathrm{TT})$ : exciton diffusion to the interface. In blend films this is to a $\mathrm{PC}_{71} \mathrm{BM}$-rich phase; in neat films diffusion is to grain boundaries or other defect sites (see above).

To determine qualitatively the contribution of ${ }^{1}(\mathrm{TT})$ and free triplets to the charge yield, we focus on Fig. 8f. Although the yield of free triplets varies significantly from 50 to $300 \mathrm{~K}$, the charge yield remains constant. If charges were only generated from free triplets, their yield should track the free triplet yield. As this is not 

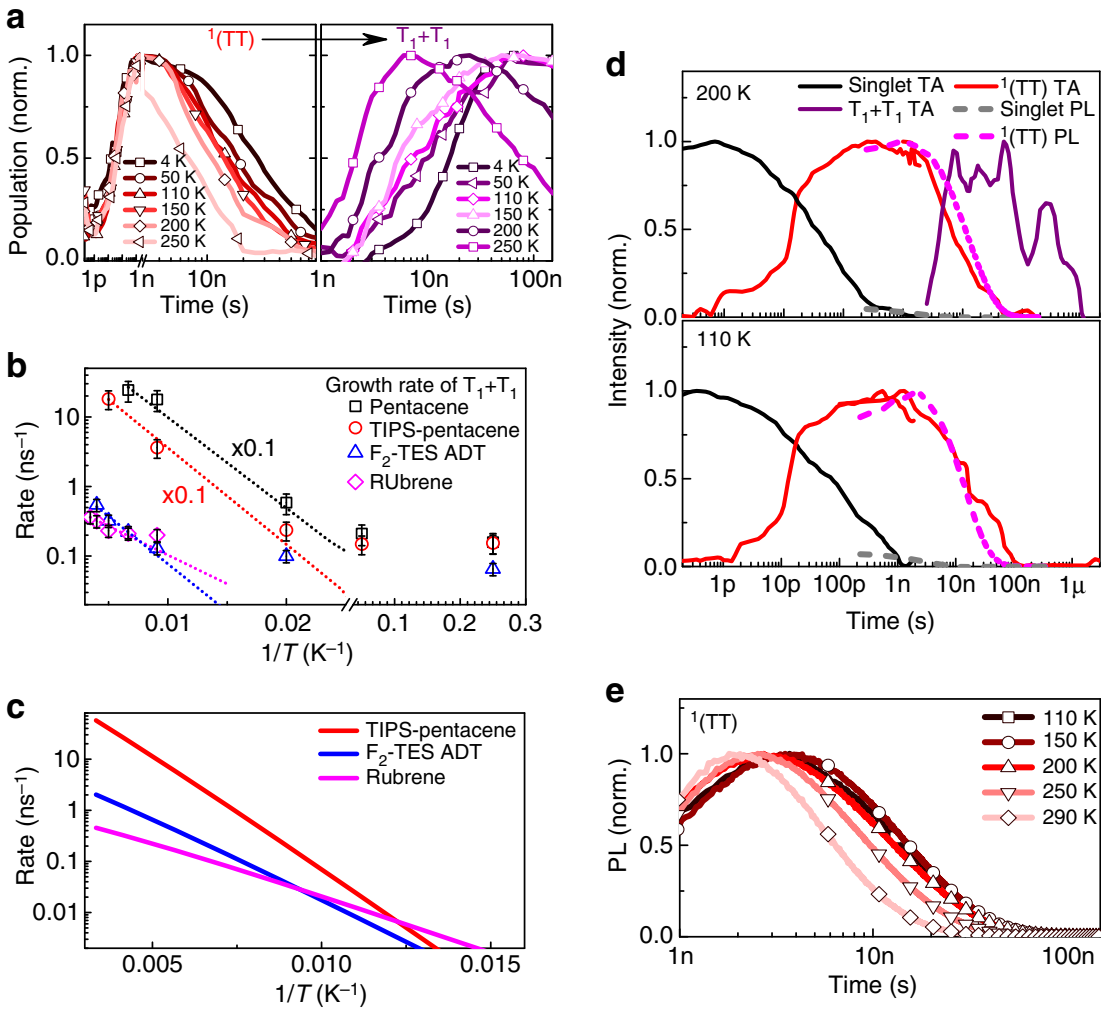

Figure 7 | 1 (TT) decay through activated triplet hopping. (a) Kinetics of ${ }^{1}(T T)$ and free triplet species in $F_{2}$-TES ADT films, extracted from TA measurements over the temperature range $4-250 \mathrm{~K}$ and revealing temperature-dependent conversion. Kinetics presented on separate time axes for clarity. (b) Rate of ${ }^{1}(\mathrm{TT})$ decay as a function of temperature. Error bars are uncertainty in the exponential fitting of ${ }^{1}(\mathrm{TT})$ decay. Dashed lines are mono-exponential fits to the high-temperature data. Although the overall rate of ${ }^{1}(\mathrm{TT})$ separation varies widely, the temperature dependence is strikingly similar across the series (activation energy $\sim 20-40 \mathrm{meV}$ ). Data for pentacene-based materials scaled by $0.1 \times$ for clarity. (c) Marcus-Levich-Jortner rate model of ${ }^{1}$ (TT) separation for three $\pi$-stacked systems. The strong variation in rate is attributed to the triplet-transfer coupling, while the temperature dependence is primarily governed by the enthalpy of ${ }^{1}(\mathrm{TT})$ separation. (d) Normalized population kinetics of excited-state species extracted from TA measurements (solid) and PL measurements (dashed) of $\mathrm{F}_{2}$-ADT single crystal at 200 and $110 \mathrm{~K}$ (see also Supplementary Fig. 21). As in thin films, sequential conversion from $S_{1}$ to emissive ${ }^{1}(T T)$ to free triplets is observed, but ${ }^{1}(\mathrm{TT})$ separation is fully suppressed at low temperature. (e) Normalized, temperature-dependent decay kinetics of ${ }^{1}(\mathrm{TT})$ in $\mathrm{F}_{2}$-ADT single crystal. At low temperature where no free triplets are formed, the decay converges to the intrinsic ${ }^{1}(\mathrm{TT})$ lifetime.

Table 2 | Parameters for triplet hopping model from ab initio calculations.

\begin{tabular}{|c|c|c|c|c|}
\hline & $V_{\mathbf{T}-\mathbf{T}}(\mathrm{meV})$ & $\Delta H(\mathrm{meV})$ & $\Delta E(\mathrm{meV})$ at $\lambda_{\text {inter }}=50 \mathrm{meV}$ & $\lambda_{\text {intra }}(\mathrm{meV})$ \\
\hline TIPS-Pentacene & 22 & 87 & 94 & 177 \\
\hline $\mathrm{F}_{2}$-TES ADT & 33 & 67 & 68 & 195 \\
\hline Rubrene & 18 & 47 & 47 & 242 \\
\hline
\end{tabular}

the case, we find that multi-electron transfer from ${ }^{1}(\mathrm{TT})$ should be the dominant pathway at low temperature.

\section{Discussion}

We have demonstrated the general presence of the bound, entangled triplet-pair state ${ }^{1}$ (TT). An interesting property of ${ }^{1}$ (TT) is that it emits as a singlet but can transfer charge from each of its constituent triplets. We find that non-radiative decay of ${ }^{1}(\mathrm{TT})$ is a dominant loss mechanism limiting further exploitation. Similarly fast non-radiative ${ }^{1}(\mathrm{TT})$ decay has been observed in covalent acene dimers ${ }^{24,63}$, carotenoid aggregates ${ }^{62,64}$ and polymers ${ }^{56,65,66}$, and understanding the mechanism of this process and minimizing it could allow for efficient solar cells with direct charge transfer from ${ }^{1}(\mathrm{TT})$. Our work also has critical implications for TTA upconversion efficiency models ${ }^{67}$, where the implicit assumption is that the singlet-character TTA encounter complex (that is, ${ }^{1}(\mathrm{TT})$ ) converts to $S_{1}$ with unity efficiency. Instead we find that ${ }^{1}(\mathrm{TT}) \rightarrow \mathrm{S}_{1}$ conversion efficiency is determined by the competition between singlet formation, triplet-pair separation and non-radiative decay.

More broadly, we consider that the generality of our findings across singlet fission materials affords a powerful platform to study the interactions between triplet excitons and the properties of bound multiexciton states and could lead to a new material set for solid-state quantum computing applications.

\section{Methods}

Materials. $\mathrm{F}_{2}$-TES ADT was synthesized as described previously ${ }^{68} . \mathrm{PC}_{71} \mathrm{BM}$, tetracene, rubrene, TIPS-pentacene and pentacene were purchased from Sigma 
a

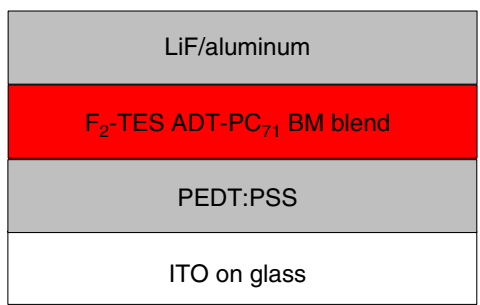

d

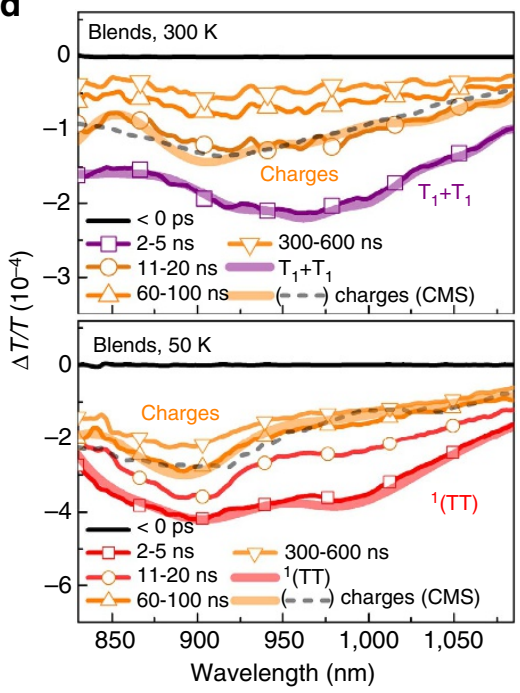

b

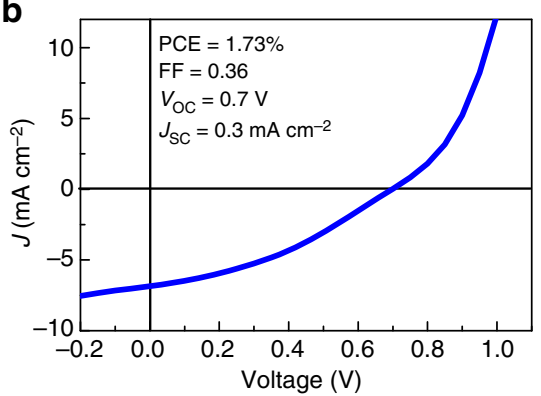

e

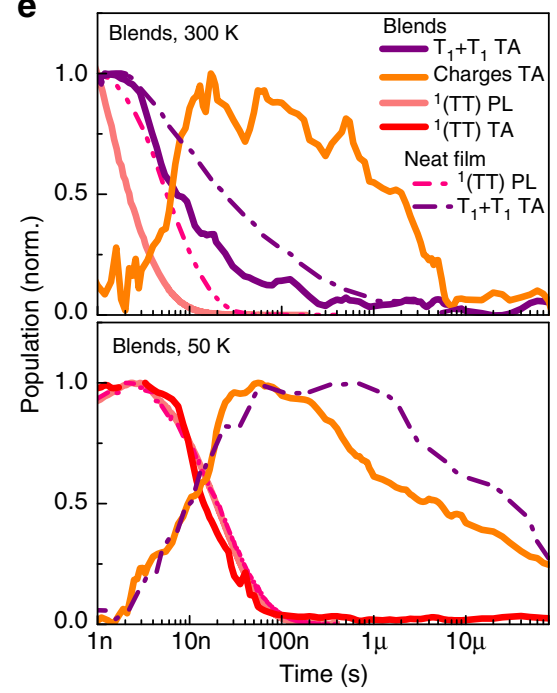

C

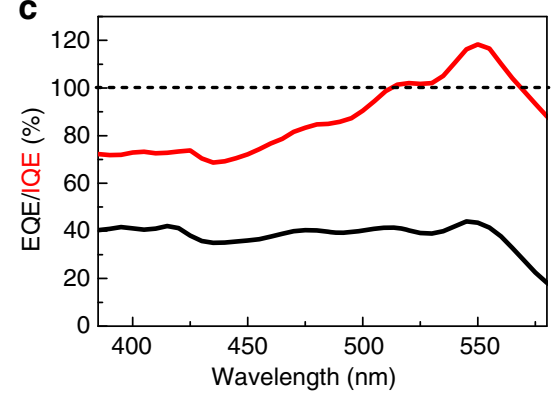

f

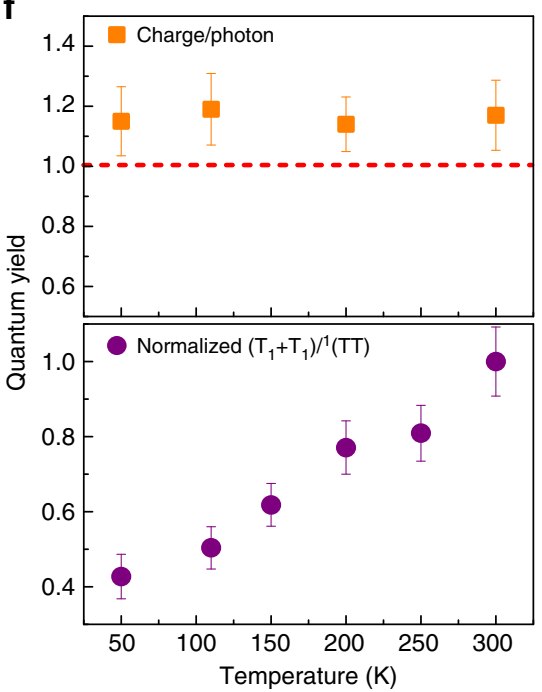

Figure $\left.8\right|^{1}(T T)$ in photovoltaic blends. (a) Schematic photovoltaic (PV) device structure. (b) Typical IV curve for $F_{2}-T E S A D T: P C C_{71} B M$ blend PV with corresponding power conversion efficiency $(P C E)$, fill-factor $(F F)$, open-circuit voltage $\left(V_{\text {oc }}\right)$ and short-circuit current $\left(J_{\text {sc }}\right)$ as indicated. (c) Despite the low PCE of $1.7 \%$, a plot of external and internal quantum efficiency (external quantum efficiency (EQE) and internal quantum efficiency (IQE) respectively) shows $>100 \%$ IQE in the region where $F_{2}$-TES ADT absorbs. (d) TA spectra of bulk-heterojunction films of $F_{2}-T E S$ ADT and PC 71 BM in 4:1 molar ratio, at $300 \mathrm{~K}$ (top) and $50 \mathrm{~K}$ (bottom) and averaged over the indicated delay ranges (thin lines). Thick lines: species extracted from spectral decomposition. The $\mathrm{F}_{2}$-TES ADT hole polaron (radical cation or 'charge') spectra extracted through spectral decomposition closely match those obtained with chargemodulation spectroscopy (CMS, dashed). (e) Triplet and charge population kinetics extracted from data in (d) and Fig. 6. (neat films, dash-dotted) and equivalent transient PL maps. Shortening of both the ${ }^{1}(\mathrm{TT})$ PL lifetime and the free triplet lifetime at $300 \mathrm{~K}$ suggest both species contribute to charge formation. At lower temperatures, no free triplets are detected prior to charge transfer at the interface. The kinetics of interfacial charge transfer closely match those of triplet-pair separation in neat films (dash-dotted). (f) Top panel: photon-to-charge conversion yield determined from TA measurements (errors from uncertainty in amplitude of TA signal and charge cross-section) of blend films reveals negligible temperature dependence. In contrast, the yield of free triplets in neat films (bottom panel, error bars reflect noise in raw TA signal), plotted as a ratio of $T_{1}+T_{1}$ to ${ }^{1}(T T)$ from TA measurements and normalized to the $300 \mathrm{~K}$ value, varies with temperature.

Aldrich. $\mathrm{F}_{2}$-TES ADT and TIPS-pentacene thin film samples were spin-cast (15 $\mathrm{mg} \mathrm{ml}^{-1}$, toluene) on polyimide precoated fused-silica substrates (the polyimide aids wetting of the organic semiconductor). For $\mathrm{F}_{2}$-TES ADT: $\mathrm{PC}_{71} \mathrm{BM}$ thin film samples, 4:1 $\mathrm{F}_{2}$-TES ADT:PC ${ }_{71} \mathrm{BM}$ blend solutions $\left(15 \mathrm{mg} \mathrm{ml}^{-1}\right.$ total material, mesitylene) were spin-cast on polyimide precoated fused-silica substrates. Samples were dried on a hot-plate at $50^{\circ} \mathrm{C}$ for $10 \mathrm{~min}$. Tetracene, rubrene and pentacene thin-film samples were prepared via thermal evaporation at a base pressure of $<6 \times 10^{-6} \mathrm{mbar}$.

Single crystals of $\mathrm{F}_{2}$-ADT were grown through a physical vapour growth method. Details of the relevant method can be seen elsewhere ${ }^{69}$. We used a growth apparatus equipped with source and growth heaters that were placed side-by-side. Temperatures of the heaters were regulated in such a way that high-quality single crystals could be produced. In the present studies we set the source and growth heaters at $240^{\circ} \mathrm{C}$ and $220^{\circ} \mathrm{C}$, respectively. The growth duration was $6 \mathrm{~h}$.

TA and PL. For TA measurements, $90 \mathrm{fs}$ pulses generated in a Ti:sapphire amplifier system (Spectra-Physics Solstice) operating at $1 \mathrm{kHz}$ were used. The broadband probe beams were generated in separate home-built non-collinear optical parametric amplifiers for visible $(500-800 \mathrm{~nm})$ and near-infrared $(800-1,100 \mathrm{~nm})$ ranges. For fs-ps TA measurements, we used either narrowband $<200 \mathrm{fs}$ pulses from a commercial travelling-wave optical parametric amplifier of superfluorescence (TOPAS) or compressed pulses ( $\sim 40 \mathrm{fs}$, spanning $525-625 \mathrm{~nm}$ ) from a home-built non-collinear optical parametric amplifier to excite the samples. The time delay between pump and probe pulses was controlled by a mechanical delay stage. Unless otherwise noted, pump intensities were kept below $5 \mu \mathrm{J} \mathrm{cm}^{-2}$ to avoid bimolecular singlet-singlet annihilation effects and the pump and probe polarization were set to magic angle $\left(54.7^{\circ}\right)$ to avoid reorientation effects. For nsTA measurements, pump pulses were generated using a frequency-doubled $Q$ switched $\sim 500 \mathrm{ps} \mathrm{Nd}_{\mathrm{YVO}}$ laser $(532 \mathrm{~nm})$. Delay times from $1 \mathrm{~ns}$ to $1 \mathrm{~ms}$ were achieved using an electronic delay generator. For time-resolved PL measurements to generate spectral maps, the samples were excited by 40 ps pulses (excitation wavelength: $470 \mathrm{~nm}$ ) operating at a repetition rate of $2.5 \mathrm{MHz}$. PL decay dynamics were resolved using electronic gating through time-correlated single-photon counting with a temporal resolution of $180 \mathrm{ps}$ and detection based on a cooled microchannel plate photomultiplier tube coupled to a monochromator. PL quantum beating measurements were performed on a separate time-correlated single photon counting system (Becker-Hickl module) system, using $100 \mathrm{fs}$ pulses at $500 \mathrm{~nm}$ with a repetition rate of $80 \mathrm{MHz}$. Here, the emission was detected with a silicon single-photon avalanche diode, yielding a temporal resolution of around 40 ps. For all solid-state time-resolved optical measurements, samples were kept at a fixed temperature in a cryostat with dynamic helium gas flow. For solution measurements, a fused silica $1 \mathrm{~mm}$ path length cuvette was used.

Triplet sensitization. Blends of $\mathrm{N}$-methylfulleropyrolidine (NMFP) and $\mathrm{F}_{2}$-TES ADT in a molar ratio of 4:1 were prepared at a concentration of $1 \mathrm{mg} \mathrm{ml}^{-1}$ in chloroform. The NMFP was excited by the $355 \mathrm{~nm}$ frequency tripled output of a

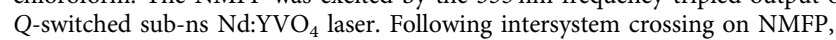


triplet transfer to $\mathrm{F}_{2}$-TES ADT occurs and is monitored by TA spectroscopy. Results are shown in Supplementary Fig. 11.

Phosphorescence. To obtain samples that gave phosphorescence, the molecule of interest was spin-coated into films doped with platinum octaethylporphyrin (purchased from Sigma Aldrich). The weight ratio of target molecules to platinum octaethylporphyrin in solution was varied from 95:5 to 98:2, to give the clearest phosphorescence signal. Mixtures were spin-coated on Spectrosil at $800-1,200$ r.p.m. for 1-2 min in a nitrogen-filled glove box and the films were then annealed at $60^{\circ} \mathrm{C}$ for $30 \mathrm{~min}$. The final samples were encapsulated to prevent sample degradation and triplet quenching by oxygen. Phosphorescence was detected using a calibrated infrared InGaAs photodiode array (ANDOR iDus 490A) coupled to a spectrograph (ANDOR Shamrock), with CW excitation at $532 \mathrm{~nm}(\sim 0.5 \mathrm{~mW})$

Optically detected magnetic resonance. Optically detected magnetic resonance (ODMR) experiments were performed to investigate the presence of free triplets in $\mathrm{F}_{2}$-TES ADT. In these measurements, the change in PL is monitored under magnetic resonance. ODMR gives a direct way of identifying triplet excitons, as the dipole-dipole interaction between electron and hole spins within the triplet exciton gives rise to characteristically broad ODMR spectra, governed by the zero-field splitting Hamiltonian $\hat{H}_{Z F S} / \hbar=D^{*}\left(\hat{S}_{Z}^{2}-\hat{\mathbf{S}}^{2} / 3\right)+E^{*}\left(\hat{S}_{X}^{2}-\hat{S}_{Y}^{2}\right)>$, where $\hat{\mathbf{S}}=\left(\hat{S}_{X}, \hat{S}_{Y}, \hat{S}_{Z}\right)$ is the triplet spin operator, and $D^{*}$ and $E^{*}$ are the zero-field splitting parameters ${ }^{70}$

Films were placed on a microwave stripline operated at a frequency of $6.2 \mathrm{GHz}$ and mounted inside an optically accessible cryostat magnet, providing the static magnetic field $B$. Excitation was provided by a $532 \mathrm{~nm}$ laser with variable intensity I. The integrated PL was collected by a photodetector, after removing the laser line with a $550 \mathrm{~nm}$ long-pass filter. Microwaves were square-wave modulated at frequency $f_{M}$, and the change in PL due to microwave transitions $\Delta \mathrm{PL}$ was recorded by monitoring the photodetector response at this microwave chopping frequency using lock-in detection.

Structural characterization. Specular X-ray diffraction was performed with a PANalytical Empyrean system in Bragg-Brentano geometry using a sealed copper tube $(\lambda=1.5418 \AA, 40 \mathrm{kV}, 40 \mathrm{~mA})$ and an X'Celerate detector operating in a one-dimensional mode. A variable slit optics choosing an illuminated length of $10 \mathrm{~mm}$ was used in combination with $0.04^{\circ}$ Soller slits at the primary as well as at the secondary side. Low temperatures down to $85 \mathrm{~K}$ were reached with the low temperature attachment TTK600 from Anton Paar Ltd using vacuum $\left(2 \times 10^{-5}\right.$ bar) conditions. Starting from room temperature the samples were cooled down in steps of $25 \mathrm{~K}$ with a cooling rate of $5 \mathrm{~K} \mathrm{~min}^{-1}$. After reaching a temperature of $85 \mathrm{~K}$, the sample was rapidly heated up to room temperature and cooled down again in steps to a temperature of $230 \mathrm{~K}$, to confirm the measurements were reproducible after thermal cycling. Careful alignment of the sample height and sample tilt were made to obtain reliable results. Peak parameters were determined by a fit of a Gaussian curve to the experimentally observed Bragg peaks.

For measurements conducted at room temperature, the out-of-plane film structure was investigated with X-ray reflectivity in a lab setup (D8, Bruker) using a wavelength of $1.5406 \AA$.

Photovoltaic device fabrication and characterization. Solar cells were fabricated on $10 \mathrm{~mm} \times 15 \mathrm{~mm}$ indium tin oxide-coated glass substrates that served as the anode. The substrates were ultrasonically cleaned in detergent, deionized water, acetone and isopropanol. A layer of $30 \mathrm{~nm}$ PEDOT:PSS (poly(3,4-ethylenedioxythiophene):poly(styrene sulphonate) (Clevios $\mathrm{PH} 1000)$ was spin-coated onto the indium tin oxide substrate and dried in air at $120^{\circ} \mathrm{C}$ for $10 \mathrm{~min}$. Fifteen milligrams of 4:1 $\mathrm{F}_{2}$-TES ADT/PC71BM blend was dissolved in $1 \mathrm{ml}$ of mesitylene and spin-coated on top of the PEDOT layer at 1,500 r.p.m. and annealed for $10 \mathrm{~min}$ at $80^{\circ} \mathrm{C}$. Finally, the $\mathrm{LiF} / \mathrm{Al}$ cathode $(50 \mathrm{~nm})$ was vacuum-evaporated onto the annealed photoactive layer. All devices were encapsulated before testing.

A $100 \mathrm{~W}$ tungsten halogen lamp (500-1,500 nm) and a $120 \mathrm{~W}$ Xenon lamp $(350-500 \mathrm{~nm}$ ) dispersed through a monochromator (Oriel Cornerstone 260) were used for external quantum efficiency measurements. For wavelengths between 375 and $900 \mathrm{~nm}$, a set of silicon diodes (ThorLabs SM05PD1A) were used. A Keithley 2635 source measure unit was used to measure the short-circuit current as a function of wavelength. The incident light was focused to a spot size of ca. $1 \mathrm{~mm}^{2}$ using a set of lenses to illuminate individual pixels of size $0.08 \mathrm{~cm}^{2}$. The current-voltage $(I-V)$ characteristics of the devices were measured under standard AM 1.5G conditions using an Abet Sun 2000 solar simulator, at an intensity equivalent to $100 \mathrm{~mW} \mathrm{~cm}^{-2}$. Spectral mismatch correction was performed before the measurements. The dark and bright $I-V$ characteristics were measured using the Keithley 2635 source measure unit.

Internal quantum efficiency plots were calculated from external quantum efficiency $(\lambda) / A(\lambda)$, where $A$ is the absorbance of the photoactive layer, which was computed with a transfer matrix approach, where $A$ was modelled according to published literature procedures ${ }^{71}$. The required values for the refractive index $n$ and the extinction coefficient $k$ were determined via ellipsometry, see Supplementary Fig. 25. The real and imaginary part of the complex refractive index were determined using variable angle spectroscopic ellipsometry (M-2000, Woollam Co.) using an optically isotropic layered optical model. The thickness was determined by fitting the data in the transparent wavelength range using the Cauchy model to describe the real part of the complex refractive index, assuming the imaginary part is zero. Film thicknesses were further determined via AFM.

Spectral decomposition techniques. To identify the spectral species present in the TA measurements and to determine their individual evolution over time, we used a combination of singular value decomposition and a spectral deconvolution $\operatorname{code}^{45}$. This code generates a given number of spectra that best reproduce the original data, while satisfying basic physical constraints such as spectral shape and population dynamics. The optimization of these spectra is done by a genetic algorithm. Once the code has minimized the residual between the obtained spectra and the original data the output is a set of spectra and kinetics for the species identified. The advantage of this optimization method over other approaches is that the genetic algorithm starts from random initial spectra and does not require a starting kinetic scheme, such as in global analysis methods. In brief, the genetic algorithm is an example of an evolutionary algorithm that factors TA spectra into a pre-determined number of spectral components. The idea is based on natural selection of genes in a population; random spectra or parents are mixed until they give separate spectra that best reproduce the original data, which constitutes a test for genetic fitness. The advantages of a genetic algorithm for our purpose over other optimization methods, such as gradient search methods, is that it is more capable of modelling a multidimensional data space that can be noisy and contain several overlapping features. Gradient search methods, on the other hand, are much more likely to get stuck in local minima.

Here, this optimization code was used to analyse the separate spectral components of TA and PL spectra. Singular value decomposition was used to give an initial estimation of the number of principal components in each spectrum. From here the optimization code was run to look for the same number of species We assumed that the initially photogenerated species primarily contains only singlets that evolve into other excited states at later time. Once it was determined whether two or three species best fit the data, the genetic algorithm was run several times on the same TA measurement to assess the reproducibility of the results. Unless otherwise mentioned, for fs-ps TA measurements, the algorithm was run over $200 \mathrm{fs}$ to $2 \mathrm{~ns}$. The precise dynamics of the sub-200 fs region are hard to assess due to the numerous fluctuations in the early-time signal and a time resolution of $\sim 200$ fs. For ns-TA measurements, the algorithm was run over 3-2,000 ns, omitting the instrument response region. For ps-PL measurements, the algorithm was run over $200 \mathrm{ps}-20 \mathrm{~ns}$.

We assess the reliability of the results of the optimization in three ways: (1) by testing the reproducibility of the results from $>10$ runs of the genetic algorithm data, as shown in Supplementary Fig. 26; (2) by comparing the spectra obtained from the optimization to the raw data obtained from both TA and PL measurements; and (3) by comparing the kinetics of population decay and/or spectra with independent measurements, for example, comparing the singlet and ${ }^{1}$ (TT) intermediate state kinetics in the TA measurements with that in the transient PL measurements. These two measurements are both conducted in the linear regime at similar excitation fluence to ensure the same photophysics are observed in both measurements.

$\boldsymbol{A} \boldsymbol{b}$ initio calculations. PL from the triplet pair state: to clarify the origin of ${ }^{1}(\mathrm{TT})$ PL, we analyse the bright exciton component in the ${ }^{1}$ (TT) state based on $a b$ initio excited-state calculations. The ${ }^{1}(\mathrm{TT})$ states of $\pi$-stacked dimers are calculated using the complete active space self-consistent field (CASSCF) method, where the active orbitals consist of the highest-occupied molecular-orbital and lowest-unoccupied molecular-orbital of two monomers, that is CASSCF $(4,4)$. The doubly excited ${ }^{1}$ (TT) diabatic state is optically dark and thus the ${ }^{1}$ (TT) PL necessitates mixing with singly excited bright exciton states. Yet, as found for the acene crystals ${ }^{16}$, the bright exciton component to the adiabatic ${ }^{1}(\mathrm{TT})$ states completely vanishes due to the relative phase factor of the wavefunctions in $\mathrm{H}$-aggregates. In this case, the symmetric ${ }^{1}$ (TT) mixes only with the dark exciton and charge transfer states, which do not contribute to PL. However, the bright exciton can be mixed with ${ }^{1}$ (TT) via symmetry-breaking effects due to intra-molecular vibronic coupling. We model this in the simplest way possible by displacing the geometry of one of monomers from the triplet minimum to the ground state minimum, (see Fig. $2 \mathrm{f}$ in the main text for results and discussion). Such a scenario is consistent with intensity borrowing induced by coupling to vibrational modes, that is, the Herzberg-Teller mechanism.

Triplet pair separation rate: we analysed the transfer rate from the bound ${ }^{1}(\mathrm{TT})$ to free triplets based on the Marcus-Levich-Jortner rate theory parameterized against $a b$ initio calculations. The electronic coupling for the intermolecular triplet transfer, $V_{\mathrm{T}-\mathrm{T}}$, (see Fig. $5 \mathrm{c}$ in the main text) was estimated using time-dependent density functional theory as applied to $\pi$-stacked dimer models. The intramolecular reorganization energy, $\lambda_{\text {intra }}$, for the triplet diffusion was computed on the monomers at the DFT level. The enthalpy change, $\Delta H$, for the ${ }^{1}(\mathrm{TT})$ separation was obtained from CASSCF $(4,4) /$ CASPT2 calculations on $\pi$-stacked trimer models, with the monomers adopting either the fully relaxed triplet geometry $(T)$ or the ground-state geometry $(G)$. Thus, in this notation, the bound and separated triplet pairs correspond to TTG and TGT configurations, respectively. 

The
theory:

$$
K_{\mathrm{TT}}=\frac{2 \pi}{\hbar} \frac{V_{\mathrm{TT}}^{2}}{\sqrt{4 \pi \lambda_{\text {inter }} k T}}\langle 0 \mid 0\rangle_{\text {intra }}^{2} \exp \left(-\frac{\left(\Delta H+\lambda_{\text {inter }}\right)^{2}}{4 \lambda_{\text {inter }} k T}\right) \exp \left(\frac{\Delta S}{k}\right)
$$

where $k, T$ and $S$ are the Boltzmann constant, temperature and entropy change, respectively. The Franck-Condon factor for the intramolecular effective mode, $\langle 0 \mid 0\rangle_{\text {intra }}$, is evaluated from the reorganization energy, $\lambda_{\text {intra }}$ and the frequency of $0.18 \mathrm{eV}$ corresponding to the usual breathing/stretching mode in aromatic/ conjugated molecules. The low-frequency intermolecular reorganization energy, $\lambda_{\text {inter }}$, is small for covalent excitations such as triplets and its calculation cumbersome. Here we take a conservative value of $0.05 \mathrm{eV}$; modifying it in a reasonable range (up to a factor of 3 ) hardly affects the conclusions below. eW account for $\Delta S$ considering the numbers of spatial and spin configurations for the bound ${ }^{1}(\mathrm{TT})$ and separated triplets. The ${ }^{1}$ (TT) separation pathway for $\pi$-stacked TIPS-pentacene, $\mathrm{F}_{2}$-TES ADT and rubrene can be regarded as a one-dimensional chain; in this case, the spatial $\Delta S$ from the bound ${ }^{1}$ (TT) to nearest-neighbour $\mathrm{T}+\mathrm{T}$ is unity. Considering the dissipation of spin correlation, the number of spin configurations of the separated T-T $(3 \times 3)$ is three times as many as the total-singlet ${ }^{1}(\mathrm{TT})$, that is $\exp (\Delta S / k)=3$.

The calculated $\Delta H$ indicates that the ${ }^{1}(\mathrm{TT})$ separation is endergonic for the three molecular materials investigated (see Fig. $5 \mathrm{~d}$ in the main text). The bound ${ }^{1}$ (TT) is stabilized by orbital delocalization as well as the admixture of singly excited electronic configurations into the nearest-neighbour TT pairs (TTG), both contributions vanishing in the case of separated triplets (TGT). The free-energy stabilization going from TGT to TTG completely dominates the activation energy $\Delta E$. Although these are overestimated by the calculations, the predicted trends in the $\Delta E$ values (namely larger in TIPS-pentacene compared to rubrene) match reasonably well with the measurements. It is interesting to point out that for rubrene in its equilibrium crystal structure at $0 \mathrm{~K}$, the singlet-triplet mixing is strictly null because of symmetry ${ }^{20}$; thus, the finite (yet smaller) $\Delta E$ value computed in that case reflects only the energy stabilization associated with direct wavefunction overlap between neighbouring triplets. The relatively large ${ }^{1}(\mathrm{TT})$ separation rate of TIPS-pentacene compared with $\mathrm{F}_{2}$-TES ADT and rubrene is rationalized by the difference in electronic coupling for triplet diffusion. Thus, based on these theoretical results, we believe the measured activation energies reported in Fig. 5 essentially reflect the energy stabilization of bound vs free triplet pairs, rather than the reorganization energy for the migration of free triplets. This is borne out by the fact that measurements in anthracene single crystals have demonstrated that the triplet diffusion coefficient is quasi temperatureindependent in the range $100-300 \mathrm{~K}$ (ref. 72) (see also references therein).

Data availability. The data that support the findings shown in both the main figures and Supplementary Figures in this study are available with the identifier https://doi.org/10.17863/CAM.9032.

\section{References}

1. Johnson, R. C. \& Merrifield, R. E. Effects of magnetic fields on the mutual annihiliation of triplet excitons in anthracene crystals. Phys. Rev. B 1, 896-902 (1970).

2. Congreve, D. N. et al. External quantum efficiency above $100 \%$ in a singlet-exciton-fission-based organic photovoltaic cell. Science 340, 334-337 (2013).

3. Bakulin, A. A. et al. Real-time observation of multiexcitonic state in ultrafast singlet fission using coherent 2D electronic spectroscopy. Nat. Chem. 8, 16-23 (2016).

4. Wan, Y. et al. Cooperative singlet and triplet exciton transport in tetracene crystals visualized by ultrafast microscopy. Nat. Chem. 7, 785-792 (2015).

5. Park, Y. I., Lee, T., Suh, D. \& Hyeon, T. Upconverting nanoparticles: a versatile platform for wide-field two-photon microscopy and multi-modal in vivo imaging. Chem. Soc. Rev. 44, 1302-1317 (2015).

6. Wang, W. et al. Efficient triplet triplet annihilation-based upconversion for nanoparticle phototargeting. Nano Lett. 15, 6332-6338 (2015).

7. Zhou, Y. et al. An upconverted photonic nonvolatile memory. Nat. Commun. 5, 4720 (2014).

8. Singh-Rachford, T. N. \& Castellano, F. N. Photon upconversion based on sensitized triplet-triplet annihilation. Coord. Chem. Rev. 254, 2560-2573 (2010).

9. Kondakov, D. Y. Triplet triplet annihilation in highly efficient fluorescent organic light-emitting diodes: current state and future outlook. Philos. Trans. A 373, 20140321 (2015).

10. Teichen, P. E. \& Eaves, J. D. Collective aspects of singlet fission in molecular crystals. J. Chem. Phys. 143, 44118 (2015).

11. Scholes, G. D. Correlated pair states formed by singlet fission and exciton-exciton annihilation. J. Phys. Chem. A 119, 12699-12705 (2015).

12. Zimmerman, P. M., Zhang, Z. \& Musgrave, C. B. Singlet fission in pentacene through multi-exciton quantum states. Nat. Chem. 2, 648-652 (2010).
13. Zimmerman, P. M., Bell, F., Casanova, D. \& Head-Gordon, M. Mechanism for singlet fission in pentacene and tetracene: from singlet exciton to two triplets. J. Am. Chem. Soc. 133, 19944-19952 (2011).

14. Feng, X., Luzanov, A. V. \& Krylov, A. I. Fission of entangled spins: an electronic structure perspective. J. Phys. Chem. Lett. 4, 3845-3852 (2013).

15. Smith, M. B. \& Michl, J. Recent advances in singlet fission. Annu. Rev. Phys. Chem. 64, 361-386 (2013).

16. Beljonne, D., Yamagata, H., Brédas, J. L., Spano, F. C. \& Olivier, Y. Charge-transfer excitations steer the davydov splitting and mediate singlet exciton fission in pentacene. Phys. Rev. Lett. 110, 226402 (2013).

17. Casanova, D. Electronic structure study of singlet fission in tetracene derivatives. J. Chem. Theory Comput. 10, 324-334 (2014).

18. Berkelbach, T. C., Hybertsen, M. S. \& Reichman, D. R. Microscopic theory of singlet exciton fission. II. Application to pentacene dimers and the role of superexchange. J. Chem. Phys. 138, 114103 (2013).

19. Aryanpour, K., Shukla, A. \& Mazumdar, S. Theory of singlet fission in polyenes, acene crystals, and covalently linked acene dimers. J. Phys. Chem. C 119, 6966-6979 (2015).

20. Tamura, H., Huix-Rotllant, M., Burghardt, I., Olivier, Y. \& Beljonne, D. First-principles quantum dynamics of singlet fission: coherent versus thermally activated mechanisms governed by molecular $\pi$ stacking. Phys. Rev. Lett. 115, 107401 (2015)

21. Stern, H. L. et al. Identification of a triplet pair intermediate in singlet exciton fission in solution. Proc. Natl Acad. Sci. USA 112, 7656-7661 (2015).

22. Herz, J. et al. Unveiling singlet fission mediating states in TIPS-pentacene and its Aza-derivatives. J. Phys. Chem. A 119, 6602-6610 (2015).

23. Korovina, N. V. et al. Singlet fission in a covalently linked cofacial alkynyltetracene dimer. J. Am. Chem. Soc. 38, 617-627 (2016).

24. Sanders, S. N. et al. Exciton correlations in intramolecular singlet fission. J. Am. Chem. Soc. 138, 7289-7297 (2016).

25. Pensack, R. D. et al. Observation of two triplet-pair intermediates in singlet exciton fission. J. Phys. Chem. Lett. 7, 2370-2375 (2016).

26. Stern, H. et al. Ultrafast triplet pair formation and subsequent thermally activated dissociation control efficient endothermic singlet exciton fission. arXiv:1704.01695 (2016).

27. Margulies, E. et al. Enabling singlet fission by controlling intramolecular charge transfer in $\pi$-stacked covalent terrylenediimide dimers. Nat. Chem. 8 , 1120-1125 (2016).

28. Tayebjee, M. et al. Quintet multiexciton dynamics in singlet fission. Nat. Phys. 13, 182-188 (2016)

29. Weiss, L. et al. Strongly exchange-coupled triplet pairs in an organic semiconductor. Nat. Phys. 13, 176-181 (2016).

30. Monahan, N. et al. Dynamics of the triplet-pair state reveals the likely coexistence of coherent and incoherent singlet fission in crystalline hexacene. Nat. Chem. 9, 341-346 (2016).

31. Bayliss, S. L. et al. Spin signatures of exchange-coupled triplet pairs formed by singlet fission. Phys. Rev. B 119, 045204 (2016).

32. Yago, T., Ishikawa, K., Katoh, R. \& Wakasa, M. Magnetic field effects on triplet pair generated by singlet fission in an organic crystal: application of radical pair model to triplet pair. J. Phys. Chem. 120, 27858-27870 (2016).

33. Chien, A. \& Zimmerman, P. Recovering dynamic correlation in spin flip configuration interaction through a difference dedicated approach. J. Chem Phys. 146, 014103 (2017).

34. Tayebjee, M. J. Y., Clady, R. G. C. R. \& Schmidt, T. W. The exciton dynamics in tetracene thin films. Phys. Chem. Chem. Phys. 15, 14797-14805 (2013).

35. Charlton, J. L., Dabestani, R. \& Saltiel, J. Triplet-triplet annihilation in anthracene dimerization. J. Am. Chem. Soc. 105, 3473-3476 (1983).

36. Walker, B. J., Musser, A. J., Beljonne, D. \& Friend, R. H. Singlet exciton fission in solution. Nat. Chem. 5, 1019-1024 (2013).

37. Musser, A. J. et al. Evidence for conical intersection dynamics mediating ultrafast singlet exciton fission. Nat. Phys. 11, 352-357 (2015).

38. Yost, S. R. et al. A transferable model for singlet-fission kinetics. Nat. Chem. 6, 492-497 (2014).

39. Piland, G. B., Burdett, J. J., Kurunthu, D. \& Bardeen, C. J. Magnetic field effects on singlet fission and fluorescence decay dynamics in amorphous rubrene. J. Phys. Chem. C 117, 1224-1236 (2013).

40. Wong, C. Y., Folie, B. D., Cotts, B. L. \& Ginsberg, N. S. Discerning variable extents of interdomain orientational and structural heterogeneity in solution-cast polycrystalline organic semiconducting thin films. J. Phys. Chem. Lett. 6, 3155-3162 (2015).

41. Müller, H. \& Bässler, H. Fluorescence of solid tetracene in the temperature range 4.2 to $300 \mathrm{~K}$. Chem. Phys. Lett. 36, 312-315 (1975).

42. Burdett, J. J., Gosztola, D. \& Bardeen, C. J. The dependence of singlet exciton relaxation on excitation density and temperature in polycrystalline tetracene thin films: kinetic evidence for a dark intermediate state and implications for singlet fission. J. Chem. Phys. 135, 214508 (2011).

43. Wilson, M. W. B. et al. Temperature-independent singlet exciton fission in tetracene. J. Am. Chem. Soc. 135, 16680-16688 (2013). 
44. Piland, G. B. \& Bardeen, C. J. How morphology affects singlet fission in crystalline tetracene. J. Phys. Chem. Lett. 6, 1841-1846 (2015).

45. Gélinas, S. et al. The binding energy of charge-transfer excitons localized at polymeric semiconductor heterojunctions. J. Phys. Chem. C 115, 7114-7119 (2011).

46. Lim, S. H., Bjorklund, T. G., Spano, F. C. \& Bardeen, C. J. Exciton delocalization and superradiance in tetracene thin films and nanoaggregates. Phys. Rev. Lett. 92, 107402 (2004).

47. Burdett, J. J. \& Bardeen, C. J. Quantum beats in crystalline tetracene delayed fluorescence due to triplet pair coherences produced by direct singlet fission. J. Am. Chem. Soc. 134, 8597-8607 (2012).

48. Wang, R. et al. Magnetic dipolar interaction between correlated triplets created by singlet fission in tetracene crystals. Nat. Commun. 6, 8602 (2015)

49. Wykes, M., Parambil, R., Beljonne, D. \& Gierschner, J. Vibronic coupling in molecular crystals: a Franck-Condon Herzberg-Teller model of $\mathrm{H}$-aggregate fluorescence based on quantum chemical cluster calculations Vibronic coupling in molecular crystals. J. Chem. Phys. 143, 114116 (2015).

50. Akselrod, G. M. et al. Visualization of exciton transport in ordered and disordered molecular solids. Nat. Commun. 5, 3646 (2014).

51. Anger, F. et al. Photoluminescence spectroscopy of pure pentacene, perfluoropentacene, and mixed thin films. J. Chem. Phys. 136, 054701 (2012).

52. Aoki-Matsumoto, T., Furuta, K., Yamada, T., Moriya, H. \& Mizuno, K. Exciton photoluminescence in pentacene single crystal. Int. J. Mod. Phys. B 15, 3753-3756 (2001).

53. Sprafke, J. K. et al. Belt-shaped pi-systems: relating geometry to electronic structure in a six-porphyrin nanoring. J. Am. Chem. Soc. 133, 17262-17273 (2011).

54. Tavan, P. \& Schulten, K. Electronic excitations in finite and infinite polyenes. Phys. Rev. B 36, 4337-4358 (1987).

55. Christensen, R. L., Kohler, B. E. \& Christensen, R. L. Vibronic coupling in polyenes: high resolution optical spectroscopy of 2,10-dimethylundecapentaene. J. Chem. Phys. 63, 1837 (1975).

56. Musser, A. J. et al. Activated singlet exciton fission in a semiconducting polymer. J. Am. Chem. Soc. 135, 12747-12754 (2013).

57. Smith, M. B. \& Michl, J. Singlet Fission. Chem. Rev. 110, 6891-6936 (2010).

58. Thorsmolle, V. K. et al. Morphology effectively controls singlet-triplet exciton relaxation and charge transport in organic semiconductors. Phys. Rev. Lett. 102, 17401-17404 (2009).

59. Wilson, M., Rao, A., Ehler, B. \& Friend, R. H. Singlet exciton fission in polycrystalline pentacene: from photophysics toward devices. Acc. Chem. Res. 46, 1330 (2013).

60. Bardeen, C. J. The structure and dynamics of molecular excitons. Annu. Rev. Phys. Chem. 65, 127-148 (2014).

61. Burgos, J., Pope, M., Swenberg, C. H. E. \& Albano, R. R. The triplet state energies of rubrene and diphenylisobenzofuran. Phys. Stat. Sol. 83, 249 (1977).

62. Musser, A. J. et al. The nature of singlet exciton fission in carotenoid aggregates. I. Am. Chem. Soc. 137, 5130-5139 (2015).

63. Lukman, S. et al. Tuneable singlet exciton fission and triplet-triplet annihilation in an orthogonal pentacene dimer. Adv. Func. Mat 25, 5452-5461 (2015).

64. Wang, C. \& Tauber, M. J. High-yield singlet fission in a zeaxanthin aggregate observed by picosecond resonance Raman spectroscopy. J. Am. Chem. Soc. 132, 13988-13991 (2010).

65. Busby, E. et al. A design strategy for intramolecular singlet fission mediated by charge-transfer states in donor-acceptor organic materials. Nat. Mater. 14, 426-433 (2015).

66. Sanders, S. N. et al. Singlet fission in polypentacene. Chem 1, 505 (2016).

67. Cheng, Y. et al. Kinetic analysis of photochemical upconversion by triplettriplet annihilation: beyond any spin statistical limit. J. Chem. Phys. Lett. 1795, 1 (1977).

68. Subramanian, S. et al. Chromophore fluorination enhances crystallization and stablity of soluble anthradithiophene semiconductors. J. Am. Chem. Soc. 130, 2706-2707 (2008).

69. Yamao, T., Shimizu, Y., Terasaki, K. \& Hotta, S. Organic light-emitting fieldeffect transistors operated by alternating-current gate voltages. Adv. Mater. 20, 4109-4112 (2008).
70. Weil, J. A. \& Bolton, J. R. in: Electron Paramagnetic Resonance, Chapter 6, 162-187 (Wiley, 2007).

71. Burkhard, G. F., Hoke, E. T. \& McGehee, M. D. Accounting for interference, scattering, and electrode absorption to make accurate internal quantum efficiency measurements in organic and other thin solar cells. Adv. Mater. 22, 3293-3297 (2010).

72. Grisanti, L. et al. Roles of local and nonlocal electron-phonon couplings in triplet exciton diffusion in the anthracene crystal. Phys. Rev. B 88, 035450 (2013).

73. Tomkiewicz, Y., Groff, R. P. \& Avakian, P. Spectroscopic approach to energetics of exciton fission and fusion in tetracene crystals. J. Chem. Phys. 54, 4504 (1971)

\section{Acknowledgements}

We thank the G8 Research Councils Initiative on Multilateral Research Funding (EPSRC EP/K025651; US National Science Foundation CMM1-1255494; Japanese Society for the Promotion of Science). J.C. thanks the University of Sheffield for a VC fellowship. A.J.M. J.C. and S.L.B. thank EPSRC (EP/M025330, EP/M01083X and EP/M025330). The work in Mons is supported by BELSPO through the PAI P6/27 Functional Supramolecular Systems project and by the Belgian National Fund for Scientific Research FNRS/F.R.S. D.B. is a Research Director of FNRS.

\section{Author contributions}

C.K.Y. measured most of the data presented in the manuscript. C.K.Y., A.J.M. and J.C. prepared the manuscript and analysed the data. H.S. and J.C. conceived the project and, together with A.J.M., supervised its execution. S.L.B. measured the ODMR data, performed the related calculations and helped prepare the manuscript. S.L. performed the phosphorescence measurements, O.B. prepared and measured the OPV devices, R.K.H. prepared $\mathrm{F}_{2}$-TES ADT, A.M. measured the charge-modulation spectroscopy data, R.R. measured the XRD data and M.M. prepared the $\mathrm{F}_{2}$-ADT single crystal. S.H., L.M.H. and J.E.A. supervised the measurements. H.T. and D.B. performed the calculations and helped prepare the manuscript.

\section{Additional information}

Supplementary Information accompanies this paper at http://www.nature.com/ naturecommunications

Competing interests: The authors declare no competing financial interests.

Reprints and permission information is available online at http://npg.nature.com/ reprintsandpermissions/

How to cite this article: Yong, C. K. et al. The entangled triplet pair state in acene and heteroacene materials. Nat. Commun. 8, 15953 doi: 10.1038/ncomms15953 (2017).

Publisher's note: Springer Nature remains neutral with regard to jurisdictional claims in published maps and institutional affiliations.

Open Access This article is licensed under a Creative Commons Ac Attribution 4.0 International License, which permits use, sharing,
adaptation, distribution and reproduction in any medium or format, as long as you give appropriate credit to the original author(s) and the source, provide a link to the Creative Commons license, and indicate if changes were made. The images or other third party material in this article are included in the article's Creative Commons license, unless indicated otherwise in a credit line to the material. If material is not included in the article's Creative Commons license and your intended use is not permitted by statutory regulation or exceeds the permitted use, you will need to obtain permission directly from the copyright holder. To view a copy of this license, visit http://creativecommons.org/ licenses/by/4.0/

(C) The Author(s) 2017 